Pacific

Journal of

Mathematics

\title{
MONIC REPRESENTATIONS AND GORENSTEIN-PROJECTIVE MODULES
}

XiU-Hua Luo and Pu Zhang 


\title{
MONIC REPRESENTATIONS AND GORENSTEIN-PROJECTIVE MODULES
}

\author{
XiU-Hua LuO AND Pu ZHANG \\ Dedicated to the memory of Hua Feng
}

\begin{abstract}
Given a finite-dimensional algebra $A$ over a field $k$, and a finite acyclic quiver $Q$, let $\Lambda=A \otimes_{k} k Q$, where $k Q$ is the path algebra of $Q$ over $k$. Then the category $\Lambda$-mod of $\Lambda$-modules is equivalent to the category $\operatorname{Rep}(Q, A)$ of representations of $Q$ over $A$. This yields the notion of monic representations of $Q$ over $A$. We denote the full subcategory of $\operatorname{Rep}(Q, A)$ consisting of monic representations of $Q$ over $A$ by $\operatorname{Mon}(Q, A)$. It is proved that $\operatorname{Mon}(Q, A)$ has Auslander-Reiten sequences.

The main result of this paper explicitly describes the Gorenstein-projective $\Lambda$-modules via the monic representations plus an extra condition. As a corollary, we prove the equivalence of three conditions: $A$ is self-injective; Gorenstein-projective $\boldsymbol{\Lambda}$-modules are exactly the monic representations of $Q$ over $A ; \operatorname{Mon}(Q, A)$ is a Frobenius category.
\end{abstract}

\section{Introduction}

Let $A$ be an Artin algebra, and $A$-mod the category of finitely generated left $A$-modules. A complete A-projective resolution is an exact sequence of finitely generated projective $A$-modules

$$
P^{\bullet}=\cdots \rightarrow P^{-1} \rightarrow P^{0} \stackrel{d^{0}}{\rightarrow} P^{1} \rightarrow \cdots
$$

such that $\operatorname{Hom}_{A}\left(P^{\bullet}, A\right)$ is also exact. A module $M \in A$-mod is Gorenstein-projective if there exists a complete $A$-projective resolution $P^{\bullet}$ such that $M \cong \operatorname{Ker} d^{0}$. Let $\mathcal{P}(A)$ be the full subcategory of $A$-mod of projective modules, and $\mathcal{G P}(A)$ the full subcategory of $A$-mod of Gorenstein-projective modules. Then

$$
\mathcal{P}(A) \subseteq \mathcal{G P}(A) \subseteq{ }^{\perp} A=\left\{X \in A-\bmod \mid \operatorname{Ext}_{A}^{i}(X, A)=0 \text { for all } i \geq 1\right\} .
$$

The authors were supported by the NSF of China (11271251) and the Doctoral Fund of the Ministry of Education of China (20120073110058). Pu Zhang is the corresponding author.

MSC2010: primary 16G10; secondary 16E65, 16G50, 16G60.

Keywords: representations of a quiver over an algebra, monic representations, Gorenstein-projective modules. 
It is clear that $\mathcal{G P}(A)=A$-mod if and only if $A$ is self-injective. If $A$ is of finite global dimension, $\mathcal{G P}(A)=\mathcal{P}(A)$ (but the converse is not true); and if $A$ is $a$ Gorenstein algebra (that is, inj. $\operatorname{dim}_{A} A<\infty$ and inj.dim $A_{A}<\infty$ ), then $\mathcal{G P}(A)={ }^{\perp} A$ (but the converse is not true); see, for example, [Enochs and Jenda 2000, Corollary 11.5.3]. This class of modules enjoys more stable properties than the usual projective modules (see [Auslander and Bridger 1969], where it was called a module of $G$-dimension zero); it becomes a main ingredient in the relative homological algebra [Enochs and Jenda 1995; 2000] and in the representation theory of algebras (see [Auslander and Reiten 1991a; 1991b; Beligiannis 2005; Gao and Zhang 2010; Iyama et al. 2011], for example), and plays a central role in the Tate cohomology of algebras (see [Avramov and Martsinkovsky 2002; Buchweitz 1987], for example). An important feature is that $\mathcal{G P}(A)$ is a Frobenius category with relative projective-injective objects being projective $A$-modules, and hence the stable category $\underline{\mathcal{G P}}(A)$ of $\mathcal{G P}(A)$ modulo $\mathcal{P}(A)$ is a triangulated category. By [Buchweitz 1987; Happel 1991], the singularity category of a Gorenstein algebra $A$ is triangle equivalent to $\underline{\mathcal{G P}(A)}$. Thus explicitly constructing all the Gorenstein-projective modules is a fundamental problem, and is useful to all of these applications.

On the other hand, the submodule category has been extensively studied by C. M. Ringel and M. Schmidmeier [2006; 2008a; 2008b]; see also [Simson 2007]. By [Kussin et al. 2012] it is also related to the singularity category; see also [Chen 2011]. It turns out that the category of the Gorenstein-projective modules is closely related to the submodule category (see [Li and Zhang 2010; Xiong and Zhang 2012]), or, in general, to the monomorphism category [Zhang 2011]. The present paper explores such a relation in a more general set-up.

Given a finite-dimensional algebra $A$ over a field $k$, and a finite acyclic quiver $Q$ (here "acyclic" means that $Q$ has no oriented cycles), let

$$
\Lambda=A \otimes_{k} k Q,
$$

where $k Q$ is the path algebra of $Q$ over $k$. We call $\Lambda$ the path algebra of a finite quiver $Q$ over $A$. As in the case of $A=k, \Lambda$-mod is equivalent to the category $\operatorname{Rep}(Q, A)$ of representations of $Q$ over $A$. This interpretation permits us to introduce the so-called monic representations of $Q$ over $A$. See Definition 2.2. Let $\operatorname{Mon}(Q, A)$ be the full subcategory of $\operatorname{Rep}(Q, A)$ consisting of monic representations of $Q$ over $A$. Then $\operatorname{Mon}(Q, A)$ is a resolving, functorially finite subcategory of $\operatorname{Rep}(Q, A)$, and hence has Auslander-Reiten sequences (see Theorem 3.1). The main result of this paper, Theorem 5.1, explicitly describes all the Gorenstein-projective $\Lambda$-modules, via the monic representations of $Q$ over $A$ plus an extra condition. We emphasize that here $\Lambda$ is not necessarily Gorenstein. By our main result, if we know all the Gorensteinprojective $A$-modules, we know all the Gorenstein-projective $\Lambda$-modules, and, in this way, we give an inductive construction of the Gorenstein-projective modules. 
The proof of Theorem 5.1 use induction on $\left|Q_{0}\right|$ and a description of the Gorensteinprojective modules over the triangular extension of two algebras via a bimodule which is projective in both sides (Theorem 4.1). As a corollary, we see that $A$ is self-injective if and only if $\mathcal{G P}(\Lambda)=\operatorname{Mon}(Q, A)$, and if and only if $\operatorname{Mon}(Q, A)$ is a Frobenius category (Corollary 6.1). As another corollary, if $Q$ has an arrow, $\mathcal{P}(\Lambda)=\operatorname{Mon}(Q, A)$ if and only if $\Lambda$ is hereditary (Corollary 6.3).

\section{Monic representations of a quiver over an algebra}

Throughout this section $k$ is a field, $Q$ a finite quiver, and $A$ a finite-dimensional $k$-algebra. We consider the path algebra $A Q$ of $Q$ over $A$, describe its module category, and introduce the concept of monic representations of $Q$ over $A$. In Subsections 2A-2D, $Q$ is not assumed to be acyclic if not otherwise stated.

2A. Given a finite quiver

$$
Q=\left(Q_{0}, Q_{1}, s, e\right)
$$

let $\mathcal{P}$ be the set of paths of $Q$. We write the conjunction of paths from right to left. If $p=\alpha_{l} \cdots \alpha_{1} \in \mathcal{P}$ with $\alpha_{i} \in Q_{1}, l \geq 1$, and $e\left(\alpha_{i}\right)=s\left(\alpha_{i+1}\right)$ for $1 \leq i \leq l-1$, we call $l$ the length of $p$ and denote it by $l(p)$, and define the starting vertex $s(p)=s\left(\alpha_{1}\right)$ and the ending vertex $e(p)=e\left(\alpha_{l}\right)$. We denote a vertex $i$ by $e_{i}$, and regard it as a path of length 0 , with $s\left(e_{i}\right)=i=e\left(e_{i}\right)$. Let $k Q$ be the path algebra of $Q$ over $k$. It is well-known that the category $k Q$-mod of finite-dimensional $k Q$-modules is equivalent to the category $\operatorname{Rep}(Q, k)$ of finite-dimensional representations of $Q$ over $k$; see, for example, [Ringel 1984, p. 44].

2B. Let $\Lambda=A Q$ be the free left $A$-module with basis $\mathcal{P}$. An element of $A Q$ is written as a finite sum $\sum_{p \in \mathcal{P}} a_{p} p$, where $a_{p} \in A$ and $a_{p}=0$ for all but finitely many $p$. Then $\Lambda$ is a $k$-algebra, with multiplication bilinearly given by

$$
\left(a_{p} p\right)\left(b_{q} q\right)=\left(a_{p} b_{q}\right)(p q)
$$

where $a_{p} b_{q}$ is the product in $A$, and $p q$ is the product in $k Q$. We have isomorphisms $\Lambda \cong A \otimes_{k} k Q \cong k Q \otimes_{k} A$ of $k$-algebras, and we call $\Lambda=A Q$ the path algebra of $Q$ over $A$.

For example, if $Q=\bullet \rightarrow \cdots \rightarrow \bullet$, the algebra $\Lambda$ is given by the upper triangular matrix algebra of $A$ :

$$
T_{n}(A)=\left(\begin{array}{ccccc}
A & A & \cdots & A & A \\
0 & A & \cdots & A & A \\
\vdots & \vdots & \ddots & \vdots & \vdots \\
0 & 0 & \cdots & A & A \\
0 & 0 & \cdots & 0 & A
\end{array}\right) \text {, }
$$


In general, if $Q$ is acyclic and $Q_{0}$ is labeled as $1, \ldots, n$ in such a way that $j>i$ whenever there is an arrow $\alpha: j \rightarrow i$ in $Q_{1}$, then

$$
k Q \cong\left(\begin{array}{ccccc}
k & k^{m_{21}} & k^{m_{31}} & \cdots & k^{m_{n 1}} \\
0 & k & k^{m_{32}} & \cdots & k^{m_{n 2}} \\
0 & 0 & k & \cdots & k^{m_{n 3}} \\
\vdots & \vdots & \vdots & & \vdots \\
0 & 0 & 0 & \cdots & k
\end{array}\right)_{n \times n}
$$

where $m_{j i}$ is the number of paths from $j$ to $i$ and $k^{m_{j i}}$ is the direct sum of $m_{j i}$ copies of $k$, and hence

$$
\Lambda \cong\left(\begin{array}{ccccc}
A & A^{m_{21}} & A^{m_{31}} & \cdots & A^{m_{n 1}} \\
0 & A & A^{m_{32}} & \cdots & A^{m_{n 2}} \\
0 & 0 & A & \cdots & A^{m_{n 3}} \\
\vdots & \vdots & \vdots & & \vdots \\
0 & 0 & 0 & \cdots & A
\end{array}\right)_{n \times n} .
$$

2C. By definition, a representation $X$ of $Q$ over $A$ is a datum

$$
X=\left(X_{i}, X_{\alpha}, i \in Q_{0}, \alpha \in Q_{1}\right),
$$

where $X_{i}$ is an $A$-module for each $i \in Q_{0}$ and $X_{\alpha}: X_{s(\alpha)} \rightarrow X_{e(\alpha)}$ is an A-map for each $\alpha \in Q_{1}$. It is a finite-dimensional representation if each $X_{i}$ is finitedimensional. We call $X_{i}$ the $i$-th branch of $X$. A morphism $f$ from representation $X$ to representation $Y$ is a datum $\left(f_{i}, i \in Q_{0}\right)$, where $f_{i}: X_{i} \rightarrow Y_{i}$ is an $A$-map for each $i \in Q_{0}$, such that, for each arrow $\alpha: j \rightarrow i$, the diagram

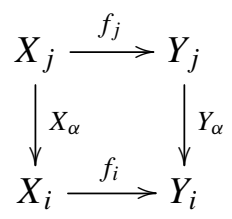

commutes. We call $f_{i}$ the $i$-th branch of $f$. If $p=\alpha_{l} \cdots \alpha_{1} \in \mathcal{P}$ with $\alpha_{i} \in Q_{1}$, $l \geq 1$, and $e\left(\alpha_{i}\right)=s\left(\alpha_{i+1}\right)$ for $1 \leq i \leq l-1$, we put $X_{p}$ to be the $A$-map $X_{\alpha_{l}} \cdots X_{\alpha_{1}}$. Denote by $\operatorname{Rep}(Q, A)$ the category of finite-dimensional representations of $Q$ over $A$. A morphism $f=\left(f_{i}, i \in Q_{0}\right)$ in $\operatorname{Rep}(Q, A)$ is a monomorphism (epimorphism, isomorphism) if and only if $f_{i}$ is injective (surjective, an isomorphism) for each $i \in Q_{0}$.

Lemma 2.1. Let $\Lambda$ be the path algebra of $Q$ over $A$. Then we have an equivalence $\Lambda-\bmod \cong \operatorname{Rep}(Q, A)$ of categories.

We omit the proof of Lemma 2.1, which is similar to the case of $A=k$; see [Auslander et al. 1995, Theorem 1.5, p. 57; Ringel 1984, p. 44]. Throughout this paper we will identify a $\Lambda$-module with a representation of $Q$ over $A$. Under this identification, a $\Lambda$-module $X$ is a representation $\left(X_{i}, X_{\alpha}, i \in Q_{0}, \alpha \in Q_{1}\right)$ of $Q$ 
over $A$, where $X_{i}=\left(1 e_{i}\right) X, 1$ is the identity of $A$, and the $A$-action on $X_{i}$ is given by $a\left(1 e_{i}\right) x=\left(1 e_{i}\right)\left(a e_{i}\right) x$ for all $x \in X$ and $a \in A$; and $X_{\alpha}: X_{s(\alpha)} \rightarrow X_{e(\alpha)}$ is the $A$-map given by the left action by $1 \alpha \in \Lambda$. On the other hand, a representation $\left(X_{i}, X_{\alpha}, i \in Q_{0}, \alpha \in Q_{1}\right)$ of $Q$ over $A$ is a $\Lambda$-module $X=\bigoplus_{i \in Q_{0}} X_{i}$, with the $\Lambda$-action on $X$ given by

$$
(a p)\left(x_{i}\right)= \begin{cases}0 & \text { if } s(p) \neq i, \\ a x_{i} & \text { if } p=e_{i}, \\ a X_{p}\left(x_{i}\right) \in X_{e(p)} & \text { if } s(p)=i \text { and } l(p) \geq 1,\end{cases}
$$

for all $a \in A, p \in \mathcal{P}, x_{i} \in X_{i}$. Let $f: X \rightarrow Y$ be a morphism in $\operatorname{Rep}(Q, A)$. Then Ker $f$ and Coker $f$ can be explicitly written out. For example, Coker $f=$ (Coker $f_{i}, \widetilde{Y}_{\alpha}, i \in Q_{0}, \alpha \in Q_{1}$ ), where, for each arrow $\alpha: j \rightarrow i$,

$$
\widetilde{Y}_{\alpha}: \text { Coker } f_{j} \rightarrow \text { Coker } f_{i}
$$

is the $A$-map induced by $Y_{\alpha}$; see (2-3). A sequence of morphisms

$$
0 \longrightarrow X \stackrel{f}{\longrightarrow} Y \stackrel{g}{\longrightarrow} Z \longrightarrow 0
$$

in $\operatorname{Rep}(Q, A)$ is exact if and only if each

$$
0 \longrightarrow X_{i} \stackrel{f_{i}}{\longrightarrow} Y_{i} \stackrel{g_{i}}{\longrightarrow} Z_{i} \longrightarrow 0
$$

is exact in $A$-mod, for $i \in Q_{0}$.

In the following, if $Q_{0}$ is labeled as $1, \ldots, n$, we also write a representation $X$ of $Q$ over $A$ as

$$
\left(\begin{array}{c}
X_{1} \\
\vdots \\
X_{n}
\end{array}\right)_{\left(X_{\alpha}, \alpha \in Q_{1}\right)}
$$

and a morphism in $\operatorname{Rep}(Q, A)$ as

$$
\left(\begin{array}{c}
f_{1} \\
\vdots \\
f_{n}
\end{array}\right)
$$

2D. The following is a central notion of this paper.

Definition 2.2. A representation $X=\left(X_{i}, X_{\alpha}, i \in Q_{0}, \alpha \in Q_{1}\right)$ of $Q$ over $A$ is $a$ monic representation, or a monic $\Lambda$-module, if, for each $i \in Q_{0}$, the $A$-map

$$
\left(X_{\alpha}\right)_{\substack{\alpha \in Q_{1} \\ e(\alpha)=i}}: \bigoplus_{\substack{\alpha \in Q_{1} \\ e(\alpha)=i}} X_{s(\alpha)} \rightarrow X_{i}
$$

is injective, or, equivalently, if the following two conditions are satisfied. 
(m1) For each $\alpha \in Q_{1}$, the map $X_{\alpha}: X_{s(\alpha)} \rightarrow X_{e(\alpha)}$ is injective.

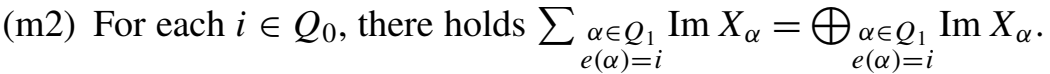

Denote by $\operatorname{Mon}(Q, A)$ the full subcategory of $\operatorname{Rep}(Q, A)$ consisting of monic representations of $Q$ over $A$. We call $\operatorname{Mon}(Q, A)$ the monomorphism category of A over $Q$.

If $Q$ is a quiver in which, for any vertex $i$, there is at most one arrow ending at $i$, condition (m2) vanishes. For example, if $Q=\bullet \rightarrow \bullet$, then $\operatorname{Mon}(Q, A)$ is called the submodule category of $A$ in [Ringel and Schmidmeier 2006; 2008a]. If

$$
Q=\bullet \rightarrow \cdots \rightarrow \bullet
$$

$\operatorname{Mon}(Q, A)$ is called the filtered chain category of $A$ in [Arnold 2000; Simson 2007].

2E. Let $Q$ be a finite acyclic quiver, $A$ a finite-dimensional algebra, and $\Lambda=$ $A \otimes_{k} k Q$. Throughout this paper, we label the vertices of $Q$ as $1,2, \ldots, n$, in such a way that if there is an arrow from $j$ to $i$, then $j>i$. Denote by $P(i)$ the indecomposable projective $k Q$-module at $i \in Q_{0}$. It is clear that $P(i) \in \operatorname{Mon}(Q, k)$; it follows that $M \otimes_{k} P(i) \in \operatorname{Mon}(Q, A)$ for $M \in A$-mod. Thus we have the functors

$$
-\otimes_{k} P(i): A-\bmod \rightarrow \operatorname{Mon}(Q, A), \quad-_{i}: \operatorname{Rep}(Q, A) \rightarrow A-\bmod
$$

(by taking the $i$-th branch).

We also need the adjoint pair $\left(-\otimes_{k} P(i),-{ }_{i}\right)$.

Lemma 2.3. For each object $X=\left(X_{i}, X_{\alpha}, i \in Q_{0}, \alpha \in Q_{1}\right) \in \Lambda$-mod and each A-module $M$, we have isomorphisms of abelian groups, which are natural in both positions

$$
\operatorname{Hom}_{\Lambda}\left(M \otimes_{k} P(i), X\right) \cong \operatorname{Hom}_{A}\left(M, X_{i}\right)
$$

for all $i \in Q_{0}$.

Proof. For $f=\left(f_{j}, j \in Q_{0}\right) \in \operatorname{Hom}_{\Lambda}\left(M \otimes_{k} P(i), X\right)$, we have $f_{i} \in \operatorname{Hom}_{A}\left(M, X_{i}\right)$. Since $M \otimes_{k} P(i)=\left(M \otimes_{k} e_{j} k Q e_{i}, \mathrm{id}_{M} \otimes \alpha, j \in Q_{0}, \alpha \in Q_{1}\right)$, it follows from the commutative diagram (2-3) that

$$
f_{j}= \begin{cases}0 & \text { if there are no paths from } i \text { to } j, \\ m \otimes_{k} p \mapsto X_{p} f_{i}(m) & \text { if there is a path } p \text { from } i \text { to } j .\end{cases}
$$

By (2-5) we see that $f \mapsto f_{i}$ gives an injective map

$$
\operatorname{Hom}_{\Lambda}\left(M \otimes_{k} P(i), X\right) \rightarrow \operatorname{Hom}_{A}\left(M, X_{i}\right) .
$$

This map is also surjective, since for a given $f_{i} \in \operatorname{Hom}_{A}\left(M, X_{i}\right), f=\left(f_{j}, j \in Q_{0}\right)$ given by (2-5) is indeed a morphism in $\operatorname{Rep}(Q, A)$ from $M \otimes_{k} P(i)$ to $X$. 
Proposition 2.4. (i) The indecomposable projective $\Lambda$-modules have the form $P \otimes_{k} P(i)$, where $P$ is an indecomposable projective A-module, and $P(i)$ is the indecomposable projective $k Q$-module at $i \in Q_{0}$.

(ii) The indecomposable projective objects in $\operatorname{Mon}(Q, A)$ are exactly the indecomposable projective $\Lambda$-modules.

(iii) If I is an indecomposable injective A-module and $P(i)$ is the indecomposable projective $k Q$-module at $i \in Q_{0}, I \otimes_{k} P(i)$ is an indecomposable injective object in $\operatorname{Mon}(Q, A)$.

Proof. (i) As a direct summand of the regular $\Lambda$-module ${ }_{\Lambda} \Lambda$, we see that $P \otimes_{k} P(i)$ is a projective $\Lambda$-module, and each projective $\Lambda$-module has this form. By (2-4) we have

$$
\operatorname{End}_{\Lambda}\left(P \otimes_{k} P(i)\right) \cong \operatorname{Hom}_{A}\left(P,\left(P \otimes_{k} P(i)\right)_{i}\right)=\operatorname{End}_{A}(P),
$$

from which we see that $P \otimes_{k} P(i)$ is indecomposable.

(ii) Note that $P \otimes_{k} P(i) \in \operatorname{Mon}(Q, A)$. By $(i)$ we know that it is an indecomposable projective object in $\operatorname{Mon}(Q, A)$. On the other hand, it is clear that $\operatorname{Mon}(Q, A)$ is closed under taking subobjects, as a consequence any indecomposable projective object in $\operatorname{Mon}(Q, A)$ has this form.

(iii) Note that $I \otimes_{k} P(i)$ is an indecomposable object in $\operatorname{Mon}(Q, A)$. Put $L=$ $D\left(A_{A}\right) \otimes_{k} k Q$, where $D=\operatorname{Hom}_{k}(-, k)$. It suffices to prove that $L$ is an injective object in $\operatorname{Mon}(Q, A)$, by induction on $\left|Q_{0}\right|$. We write $L=\left(L_{i}, L_{\alpha}, i \in Q_{0}, \alpha \in Q_{1}\right)$.

Let $Q^{\prime}$ be the quiver obtained from $Q$ by deleting a sink vertex $1, L^{\prime}$ the representation in $\operatorname{Rep}\left(Q^{\prime}, A\right)$ obtained from $L$ by deleting the branch $L_{1}$. We observe that $L^{\prime}=D\left(A_{A}\right) \otimes_{k} k Q^{\prime}$, and by inductive hypothesis $L^{\prime}$ is an injective object in $\operatorname{Mon}\left(Q^{\prime}, A\right)$.

Let $0 \rightarrow X \stackrel{f}{\longrightarrow} Y \stackrel{g}{\longrightarrow} Z \rightarrow 0$ be an exact sequence in $\operatorname{Mon}(Q, A)$, with $X=\left(X_{i}, X_{\alpha}, i \in Q_{0}, \alpha \in Q_{1}\right)$, and $h: X \rightarrow L$ a morphism in $\operatorname{Rep}(Q, A)$. Let $X^{\prime}$ be the representation in $\operatorname{Rep}\left(Q^{\prime}, A\right)$ obtained from $X$ by deleting the branch $X_{1}$, and similarly for $Y^{\prime}, Z^{\prime}$. Then we have an exact sequence

$$
0 \longrightarrow X^{\prime} \stackrel{f^{\prime}}{\longrightarrow} Y^{\prime} \stackrel{g^{\prime}}{\longrightarrow} Z^{\prime} \longrightarrow 0
$$

in $\operatorname{Mon}\left(Q^{\prime}, A\right)$, where $f^{\prime}$ is the morphism in $\operatorname{Rep}\left(Q^{\prime}, A\right)$ obtained from $f$ by deleting the branch $f_{1}$, and similarly for $g^{\prime}$ and for $h^{\prime}: X^{\prime} \rightarrow L^{\prime}$. Since $L^{\prime}$ is an injective object in $\operatorname{Mon}\left(Q^{\prime}, A\right)$, by definition we have a morphism

$$
u^{\prime}=\left(\begin{array}{c}
u_{2} \\
\vdots \\
u_{n}
\end{array}\right): Y^{\prime} \rightarrow L^{\prime}
$$


in $\operatorname{Rep}\left(Q^{\prime}, A\right)$ such that $h^{\prime}=u^{\prime} f^{\prime}$. It suffices to construct an $A$-map

such that $u=\left(\begin{array}{c}u_{1} \\ u_{2} \\ \vdots \\ u_{n}\end{array}\right): Y \rightarrow L$ is a morphism in $\operatorname{Rep}(Q, A)$, and that $h_{1}=u_{1} f_{1}$.

First, we have an $A$-map $u_{1}^{\prime}: Y_{1} \rightarrow L_{1}$ such that the diagram

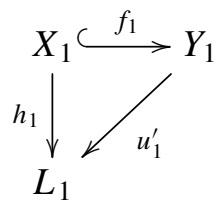

commutes. Consider the A-map

$$
\left(L_{\alpha} u_{s(\alpha)}-u_{1}^{\prime} Y_{\alpha}\right)_{\substack{\alpha \in Q_{1} \\ e(\alpha)=1}} \bigoplus_{\substack{\alpha \in Q_{1} \\ e(\alpha)=1}} Y_{s(\alpha)} \rightarrow L_{1} .
$$

Since we have the exact sequence of $A$-modules

$$
0 \longrightarrow \bigoplus_{\substack{\alpha \in Q_{1} \\ e(\alpha)=1}} X_{s(\alpha)} \stackrel{\operatorname{diag}\left(f_{s(\alpha)}\right)}{\longrightarrow} \bigoplus_{\substack{\alpha \in Q_{1} \\ e(\alpha)=1}} Y_{s(\alpha)} \stackrel{\operatorname{diag}\left(g_{s(\alpha)}\right)}{\longrightarrow} \bigoplus_{\substack{\alpha \in Q_{1} \\ e(\alpha)=1}} Z_{s(\alpha)} \longrightarrow 0
$$

and since

$$
\begin{aligned}
\left(L_{\alpha} u_{s(\alpha)}-u_{1}^{\prime} Y_{\alpha}\right)_{\substack{\alpha \in Q_{1} \\
e(\alpha)=1}} \circ \operatorname{diag}\left(f_{s(\alpha)}\right) & =\left(L_{\alpha} u_{s(\alpha)} f_{s(\alpha)}-u_{1}^{\prime} Y_{\alpha} f_{s(\alpha)}\right)_{\substack{\alpha \in Q_{1} \\
e(\alpha)=1}} \\
& =\left(L_{\alpha} u_{s(\alpha)} f_{s(\alpha)}-u_{1}^{\prime} f_{1} X_{\alpha}\right)_{\substack{\alpha \in Q_{1} \\
e(\alpha)=1}} \\
& =\left(L_{\alpha} h_{s(\alpha)}-h_{1} X_{\alpha}\right)_{\substack{\alpha \in Q_{1} \\
e(\alpha)=1}} \\
& =0, \quad
\end{aligned}
$$

where the second equality follows from the fact that $f: X \rightarrow Y$ is a morphism in $\operatorname{Rep}(Q, A)$, it follows that $\left(L_{\alpha} u_{s(\alpha)}-u_{1}^{\prime} Y_{\alpha}\right)_{\alpha \in Q_{1}}$ factors through $\operatorname{diag}\left(g_{s(\alpha)}\right)$. That is, there is an $A$-map

such that

$$
v_{1}: \bigoplus_{\substack{\alpha \in Q_{1} \\ e(\alpha)=1}} Z_{s(\alpha)} \rightarrow L_{1}
$$

$$
\left(L_{\alpha} u_{s(\alpha)}-u_{1}^{\prime} Y_{\alpha}\right)_{\substack{\alpha \in Q_{1} \\ e(\alpha)=1}}=v_{1} \circ \operatorname{diag}\left(g_{s(\alpha)}\right) .
$$

Since $L_{1}$ is an injective $A$-module and

$$
\left(Z_{\alpha}\right)_{\substack{\alpha \in Q_{1} \\ e(\alpha)=1}}: \bigoplus_{\substack{\alpha \in Q_{1} \\ e(\alpha)=1}} Z_{s(\alpha)} \rightarrow Z_{1}
$$


is an injective $A$-map, it follows that there is an $A$-map $w_{1}: Z_{1} \rightarrow L_{1}$, such that $v_{1}=w_{1} \circ\left(Z_{\alpha}\right)_{\substack{\alpha \in Q_{1} \\ e(\alpha)=1}}$. So we have

$$
\left(L_{\alpha} u_{s(\alpha)}-u_{1}^{\prime} Y_{\alpha}\right)_{\substack{\alpha \in Q_{1} \\ e(\alpha)=1}}=w_{1} \circ\left(Z_{\alpha}\right)_{\substack{\alpha \in Q_{1} \\ e(\alpha)=1}} \circ \operatorname{diag}\left(g_{s(\alpha)}\right)=\left(w_{1} g_{1} Y_{\alpha}\right)_{\substack{\alpha \in Q_{1} \\ e(\alpha)=1}},
$$

where the second equality follows from the fact that $g: Y \rightarrow Z$ is a morphism in $\operatorname{Rep}(Q, A)$. This means that for each $\alpha \in Q_{1}$ with $e(\alpha)=1$ we have

$$
L_{\alpha} u_{s(\alpha)}-u_{1}^{\prime} Y_{\alpha}=w_{1} g_{1} Y_{\alpha} .
$$

Now put $u_{1}=u_{1}^{\prime}+w_{1} g_{1}: Y_{1} \rightarrow L_{1}$. Then (2-6) together with the inductive hypothesis implies that

$$
u=\left(\begin{array}{c}
u_{1} \\
u_{2} \\
\vdots \\
u_{n}
\end{array}\right): Y \rightarrow L
$$

is a morphism in $\operatorname{Rep}(Q, A)$. It is clear that

$$
u_{1} f_{1}=\left(u_{1}^{\prime}+w_{1} g_{1}\right) f_{1}=u_{1}^{\prime} f_{1}=h_{1} .
$$

This completes the proof.

2F. Recall from [Auslander and Reiten 1991a] that a full subcategory $\mathcal{X}$ of $A$-mod is resolving if $\mathcal{X}$ contains all projective $A$-modules and $\mathcal{X}$ is closed under extensions, kernels of epimorphisms, and direct summands. It is straightforward to verify that $\operatorname{Mon}(Q, A)$ is closed under extensions, kernels of epimorphisms, and direct summands. By Proposition 2.4 we have the following.

Corollary 2.5. For a finite acyclic quiver $Q$ and a finite-dimensional algebra A, $\operatorname{Mon}(Q, A)$ is a resolving subcategory of $\operatorname{Rep}(Q, A)$.

2G. There is another similar but different notion. Let $A=k Q / I$ be a finitedimensional $k$-algebra, where $I$ is an admissible ideal of $k Q$. An $I$-bounded representations of $Q$ over $k$ is a datum $X=\left(X_{i}, X_{\alpha}, i \in Q_{0}, \alpha \in Q_{1}\right)$, where $X_{i}$ is a $k$-space for each $i \in Q_{0}$, and $X_{\alpha}: X_{s(\alpha)} \rightarrow X_{e(\alpha)}$ is a $k$-linear map for each $\alpha \in Q_{1}$, such that $\sum_{p \in \mathcal{P}} c_{p} X_{p}=0$ for each element $\sum_{p \in \mathcal{P}} c_{p} p \in I$, where $l(p) \geq 2$ and $c_{p} \in k$. An $I$-bounded representation $X=\left(X_{i}, X_{\alpha}, i \in Q_{0}, \alpha \in Q_{1}\right)$ of $Q$ over $k$ is a monic representation, if for each $i \in Q_{0}$ the $k$-linear map

$$
\left(X_{\alpha}\right)_{\substack{\alpha \in Q_{1} \\ e(\alpha)=i}}: \bigoplus_{\substack{\alpha \in Q_{1} \\ e(\alpha)=i}} X_{s(\alpha)} \rightarrow X_{i}
$$

is injective. Let $\operatorname{Rep}(Q, I, k)$ be the category of finite-dimensional $I$-bounded representations of $Q$ over $k$. There is an equivalence of categories between $A$-mod 
and $\operatorname{Rep}(Q, I, k)$; see [Auslander et al. 1995, Proposition 1.7, p. 60; Ringel 1984, p. 45]. Let $\operatorname{Mon}(Q, I, k)$ denote the full subcategory of $\operatorname{Rep}(Q, I, k)$ of $I$-bounded monic representations $Q$ over $k$. Then $\operatorname{Mon}(Q, 0, k)=\operatorname{Mon}(Q, k)$.

Proposition 2.6. Let $A=k Q / I$ be a finite-dimensional $k$-algebra, where $I$ is an admissible ideal of $k Q$. Then $\mathcal{P}(A) \subseteq \operatorname{Mon}(Q, I, k)$ if and only if $A$ is hereditary.

Proof. If $A$ is hereditary, $I=0$. It is clear $\mathcal{P}(k Q) \subseteq \operatorname{Mon}(Q, 0, k)$.

Conversely, if $I \neq 0$, take an element $\sum_{p \in \mathcal{P}} c_{p} p \in I$ with $l(p) \geq 2$ and $c_{p} \in k$. We may assume that all the paths $p$ with $c_{p} \neq 0$ have the same starting vertex $j$ and the same ending vertex $i$. Consider the projective $A$-module $P(j)=A e_{j}$. As an $I$-bounded representation of $Q$ over $k$, we write $P(j)$ as

$$
P(j)=\left(e_{t} k Q e_{j}, f_{\alpha}, t \in Q_{0}, \alpha \in Q_{1}\right) .
$$

Let $\alpha_{1}, \ldots, \alpha_{m}$ be all the arrows of $Q$ ending at $i$. We claim that

$$
\left(f_{\alpha_{v}}\right)_{1 \leq v \leq m}: \bigoplus_{1 \leq v \leq m} e_{s\left(\alpha_{v}\right)} k Q e_{j} \rightarrow e_{i} k Q e_{j}
$$

is not injective, where $f_{\alpha_{v}}$ is the $k$-linear map given by the left multiplication by $\alpha_{v}$. Since each path from $j$ to $i$ must go through some $\alpha_{v}$, and $\sum_{p \in \mathcal{P}} c_{p} f_{p}=0$, it follows that

$$
\sum_{1 \leq v \leq m} \operatorname{dim}_{k}\left(e_{s\left(\alpha_{v}\right)} k Q e_{j}\right)>\operatorname{dim}_{k}\left(e_{i} k Q e_{j}\right) .
$$

This justifies the claim, that is, $P(j) \notin \operatorname{Mon}(Q, I, k)$.

Now, let $\Lambda=A \otimes_{k} k Q$ be the path algebra of $Q$ over $A$. Assume that $\Lambda$ is of the form $\Lambda=k Q^{\prime} / I^{\prime}$, where $Q^{\prime}$ is a finite quiver and $I^{\prime}$ is an admissible ideal of $k Q^{\prime}$. We emphasize that, in general,

$$
\operatorname{Mon}(Q, A) \neq \operatorname{Mon}\left(Q^{\prime}, I^{\prime}, k\right) .
$$

In fact, $\mathcal{P}(\Lambda) \subseteq \operatorname{Mon}(Q, A)$ (Proposition 2.4); but generally $\mathcal{P}(\Lambda) \subseteq \operatorname{Mon}\left(Q^{\prime}, I^{\prime}, k\right)$ is not true, as Proposition 2.6 shows. This is the reason why we do not use the notation $\operatorname{Mon}(\Lambda)$.

\section{Functorial finiteness of $\operatorname{Mon}(Q, A)$ in $\operatorname{Rep}(Q, A)$}

The aim of this section is to prove the following.

Theorem 3.1. Let $Q$ be a finite acyclic quiver, and $A$ a finite-dimensional algebra. Then $\operatorname{Mon}(Q, A)$ is functorially finite in $\operatorname{Rep}(Q, A)$ and $\operatorname{Mon}(Q, A)$ has AuslanderReiten sequences. 
The idea of the proof given below is essentially due to Ringel and Schmidmeier [2008a] for the case of $Q=\bullet \rightarrow \bullet$. The same result for the case of

$$
Q=\bullet \rightarrow \cdots \rightarrow \bullet_{1}
$$

has been obtained in [Moore 2010; Zhang 2011].

3A. Let $Q$ be a finite acyclic quiver. Remember we label the vertices of $Q$ as $1,2, \ldots, n$, such that if there is an arrow from $j$ to $i, j>i$. So vertex 1 is a sink. Denote by $\mathcal{P}(\rightarrow i)$ the set of all the paths $p$ with ending vertex $e(p)=i$ and $l(p) \geq 1$.

For $X \in \operatorname{Rep}(Q, A)$ and $i \in Q_{0}$, put $K_{i}$ to be the kernel of the $A$-map

$$
\left(X_{\alpha}\right)_{\substack{\alpha \in Q_{1} \\ e(\alpha)=i}}: \bigoplus_{\substack{\alpha \in Q_{1} \\ e(\alpha)=i}} X_{S(\alpha)} \rightarrow X_{i} .
$$

Fix an injective envelope $\delta_{i}: K_{i} \hookrightarrow I K_{i}$ of $K_{i}$. Then there is an $A$-map

$$
\left(\varphi_{\alpha}\right)_{\substack{\alpha \in Q_{1} \\ e(\alpha)=i}}: \bigoplus_{\substack{\alpha \in Q_{1} \\ e(\alpha)=i}} X_{s(\alpha)} \rightarrow I K_{i}
$$

such that the diagram

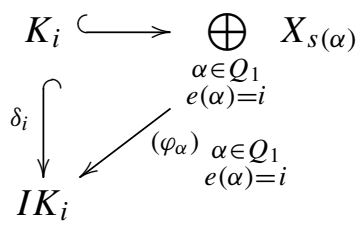

commutes for each $i \in Q_{0}$. We construct a representation

$$
\operatorname{rMon}(X)=\left(\operatorname{rMon}(X)_{i}, \operatorname{rMon}(X)_{\alpha}, i \in Q_{0}, \alpha \in Q_{1}\right) \in \operatorname{Rep}(Q, A)
$$

as follows. For each $i \in Q_{0}$, define

$$
\operatorname{rMon}(X)_{i}=X_{i} \oplus I K_{i} \oplus \bigoplus_{p \in \mathcal{P}(\rightarrow i)} I K_{s(p)} .
$$

(Note that if $i$ is a source, by definition $\operatorname{rMon}(X)_{i}=X_{i}$, and that if $p_{1}, \ldots, p_{m}$ are all the paths in $\mathcal{P}(\rightarrow i)$ with the same starting vertex $j$, the $I K_{j} \oplus \cdots \oplus I K_{j}$ is a direct summand of $\bigoplus_{p \in \mathcal{P}(\rightarrow i)} I K_{s(p)}$.)

For each arrow $\alpha: j \rightarrow i$, define

$$
\mathrm{rMon}(X)_{\alpha}: X_{j} \oplus I K_{j} \oplus \bigoplus_{p \in \mathcal{P}(\rightarrow j)} I K_{s(p)} \rightarrow X_{i} \oplus I K_{i} \oplus \bigoplus_{q \in \mathcal{P}(\rightarrow i)}^{\bigoplus I K_{s(q)}}
$$


to be the $A$-map given by

$$
x_{j}+k_{j}+\sum_{p \in \mathcal{P}(\rightarrow j)} k_{s(p)} \mapsto X_{\alpha}\left(x_{j}\right)+\varphi_{\alpha}\left(x_{j}\right)+k_{j}+\sum_{p \in \mathcal{P}(\rightarrow j)} k_{s(\alpha p)},
$$

where $x_{j} \in X_{j}, k_{j} \in I K_{j}, k_{s(p)} \in I K_{s(p)}$. Note that $s(p)=s(\alpha p)$, and that $k_{s(\alpha p)}$ is just $k_{s(p)}$. Also note that at the right side of (3-3), $k_{j}$ and $\sum_{p \in \mathcal{P}(\rightarrow j)} k_{s(\alpha p)}$ belong to different direct summands of $\bigoplus_{q \in \mathcal{P}(\rightarrow i)} I K_{s(q)}$.

Lemma 3.2. For $X \in \operatorname{Rep}(Q, A)$, we have $\operatorname{rMon}(X) \in \operatorname{Mon}(Q, A)$.

Proof. For each $i \in Q_{0}$, let $\alpha_{1}, \ldots, \alpha_{m}$ be all the arrows ending at $i$. By definition we only need to prove that the $A$-map

$$
\left(\mathrm{rMon}(X)_{\alpha_{1}}, \ldots, \mathrm{rMon}(X)_{\alpha_{m}}\right): \bigoplus_{1 \leq j \leq m} \mathrm{rMon}(X)_{s\left(\alpha_{j}\right)} \rightarrow \operatorname{rMon}(X)_{i}
$$

is injective. This is clear by (3-1)-(3-3). For completeness we include a justification.

Suppose $z_{j}=x_{s\left(\alpha_{j}\right)}+k_{s\left(\alpha_{j}\right)}+\left(\sum_{p \in \mathcal{P}\left(\rightarrow s\left(\alpha_{j}\right)\right)} k_{s(p)}\right) \in \operatorname{rMon}(X)_{s\left(\alpha_{j}\right)}, j=1, \ldots, m$, and $\sum_{1 \leq j \leq m} \operatorname{rMon}(X)_{\alpha_{j}}\left(z_{j}\right)=0$. Then by (3-3) we have

$$
\begin{array}{r}
0=\sum_{1 \leq j \leq m} X_{\alpha_{j}}\left(x_{s\left(\alpha_{j}\right)}\right)+\sum_{1 \leq j \leq m} \varphi_{\alpha_{j}}\left(x_{s\left(\alpha_{j}\right)}\right)+\sum_{1 \leq j \leq m} k_{s\left(\alpha_{j}\right)}+\sum_{1 \leq j \leq m} \sum_{p \in \mathcal{P}\left(\rightarrow s\left(\alpha_{j}\right)\right)} k_{s\left(\alpha_{j} p\right)} \\
\in X_{i} \oplus I K_{i} \oplus \bigoplus_{q \in \mathcal{P}(\rightarrow i)}^{\bigoplus I K_{s(q)} .}
\end{array}
$$

Thus

$$
\sum_{1 \leq j \leq m} X_{\alpha_{j}}\left(x_{s\left(\alpha_{j}\right)}\right)=0, \quad \sum_{1 \leq j \leq m} \varphi_{\alpha_{j}}\left(x_{s\left(\alpha_{j}\right)}\right)=0,
$$

and $k_{s\left(\alpha_{j}\right)}=0=k_{s\left(\alpha_{j} p\right)}$ for all $j=1, \ldots, m$ and all $p \in \mathcal{P}\left(\rightarrow s\left(\alpha_{j}\right)\right)$. Note that $\sum_{1 \leq j \leq m} X_{\alpha_{j}}\left(x_{s\left(\alpha_{j}\right)}\right)=0$ implies

$$
\left(\begin{array}{c}
x_{s\left(\alpha_{1}\right)} \\
\vdots \\
x_{s\left(\alpha_{m}\right)}
\end{array}\right) \in K_{i}
$$

By (3-1) we have

$$
\delta_{i}\left(\begin{array}{c}
x_{s\left(\alpha_{1}\right)} \\
\vdots \\
\left.x_{s\left(\alpha_{m}\right)}\right)
\end{array}\right)=\sum_{1 \leq j \leq m} \varphi_{\alpha_{j}}\left(x_{s\left(\alpha_{j}\right)}\right)=0 .
$$

Since $\delta_{i}$ is injective, we have $x_{s\left(\alpha_{j}\right)}=0$ for $j=1, \ldots, m$. Thus $z_{j}=0$ for $j=1, \ldots, m$. This completes the proof. 
3B. Let $\mathcal{X}$ be a full subcategory of $A$-mod. Recall from [Auslander and Reiten 1991a] that a right $\mathcal{X}$-approximation of $M$ is a morphism $f: X \rightarrow M$ with $X \in \mathcal{X}$ such that the induced homomorphism $\operatorname{Hom}_{A}\left(X^{\prime}, X\right) \rightarrow \operatorname{Hom}_{A}\left(X^{\prime}, M\right)$ is surjective for each $X^{\prime} \in \mathcal{X}$. If every object $M$ admits a right $\mathcal{X}$-approximation, $\mathcal{X}$ is called a contravariantly finite subcategory in A-mod. Dually one has the concept of $a$ covariantly finite subcategory in $A$-mod. If $\mathcal{X}$ is both contravariantly and covariantly finite in $A$-mod, $\mathcal{X}$ is a functorially finite subcategory in $A$-mod.

Proposition 3.3. Let $Q$ be a finite acyclic quiver, and $A$ a finite-dimensional algebra. Then $\operatorname{Mon}(Q, A)$ is contravariantly finite in $\operatorname{Rep}(Q, A)$.

More precisely, let $X \in \operatorname{Rep}(Q, A), f=\left(f_{i}, i \in Q_{0}\right): \operatorname{rMon}(X) \rightarrow X$, where $f_{i}: \operatorname{rMon}(X)_{i} \rightarrow X_{i}$ is the canonical projection. Then $f$ is a right $\operatorname{Mon}(Q, A)$ approximation of $X$.

Proof. We use induction to prove that $f$ is a right $\operatorname{Mon}(Q, A)$-approximation of $X$. The assertion trivially holds if $\left|Q_{0}\right|=1$. Suppose that the assertion holds for the quivers $Q$ with $\left|Q_{0}\right|=n-1$. Assume that $\left|Q_{0}\right|=n$ and that

$$
g=\left(\begin{array}{c}
g_{1} \\
\vdots \\
g_{n}
\end{array}\right): Y \rightarrow X
$$

is a morphism in $\operatorname{Rep}(Q, A)$ with $Y \in \operatorname{Mon}(Q, A)$. We need to prove that there is a morphism

$$
h=\left(\begin{array}{c}
h_{1} \\
\vdots \\
h_{n}
\end{array}\right): Y \rightarrow \mathrm{rMon}(X)
$$

in $\operatorname{Rep}(Q, A)$ such that $g=f h$.

Let $Q^{\prime}$ be the quiver obtained from $Q$ by deleting vertex $1, X^{\prime}$ the representation in $\operatorname{Rep}\left(Q^{\prime}, A\right)$ obtained from $X$ by deleting the branch $X_{1}$, and $Y^{\prime}$ the representation in $\operatorname{Mon}\left(Q^{\prime}, A\right)$ obtained from $Y$ by deleting the branch $Y_{1}$. Then by definition $\operatorname{rMon}\left(X^{\prime}\right)$ is exactly the representation in $\operatorname{Mon}\left(Q^{\prime}, A\right)$ obtained from $\operatorname{rMon}(X)$ by deleting the branch $\mathrm{rMon}(X)_{1}$. Further,

$$
\left(\begin{array}{c}
f_{2} \\
\vdots \\
f_{n}
\end{array}\right): \operatorname{rMon}\left(X^{\prime}\right) \rightarrow X^{\prime} \text { and } \quad\left(\begin{array}{c}
g_{2} \\
\vdots \\
g_{n}
\end{array}\right): Y^{\prime} \rightarrow X^{\prime}
$$

are morphisms in $\operatorname{Rep}\left(Q^{\prime}, A\right)$. By the inductive hypothesis there is a morphism

$$
\left(\begin{array}{c}
h_{2} \\
\vdots \\
h_{n}
\end{array}\right): Y^{\prime} \rightarrow \mathrm{rMon}\left(X^{\prime}\right)
$$


in $\operatorname{Rep}\left(Q^{\prime}, A\right)$, such that

$$
\left(\begin{array}{c}
g_{2} \\
\vdots \\
g_{n}
\end{array}\right)=\left(\begin{array}{c}
f_{2} \\
\vdots \\
f_{n}
\end{array}\right)\left(\begin{array}{c}
h_{2} \\
\vdots \\
h_{n}
\end{array}\right)
$$

Let $\alpha_{1}, \ldots, \alpha_{m}$ be all the arrows ending at 1 . Since

$$
\left(Y_{\alpha_{1}}, \ldots, Y_{\alpha_{m}}\right): \bigoplus_{1 \leq j \leq m} Y_{s\left(\alpha_{j}\right)} \rightarrow Y_{1}
$$

is an injective $A$-map and $I K_{1} \oplus\left(\bigoplus_{p \in \mathcal{P}(\rightarrow 1)} I K_{s(p)}\right)$ is an injective $A$-module, it follows that there is a map

$$
\eta: Y_{1} \rightarrow I K_{1} \oplus \bigoplus_{p \in \mathcal{P}(\rightarrow 1)} I K_{s(p)}
$$

such that the diagram

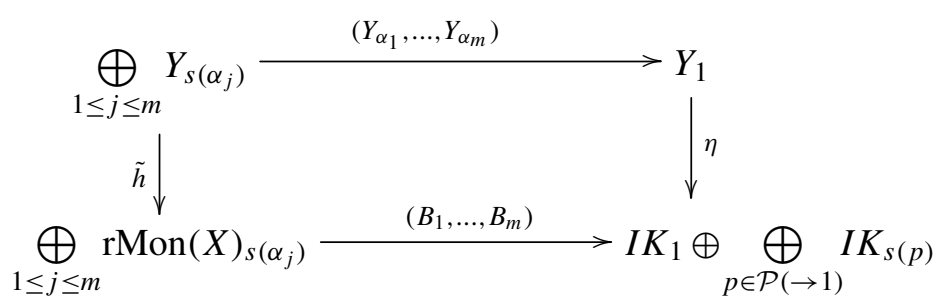

commutes, where $\tilde{h}=\operatorname{diag}\left(h_{s\left(\alpha_{1}\right)}, \ldots, h_{s\left(\alpha_{m}\right)}\right)$ and, for each $j=1, \ldots, m$,

$$
B_{j}: \operatorname{rMon}(X)_{s\left(\alpha_{j}\right)} \rightarrow I K_{1} \oplus \bigoplus_{p \in \mathcal{P}(\rightarrow 1)} I K_{s(p)}
$$

is the $A$-map given by

$$
x_{s\left(\alpha_{j}\right)}+k_{s\left(\alpha_{j}\right)}+\sum_{p \in \mathcal{P}\left(\rightarrow s\left(\alpha_{j}\right)\right)} k_{s(p)} \mapsto \varphi_{\alpha_{j}}\left(x_{s\left(\alpha_{j}\right)}\right)+k_{s\left(\alpha_{j}\right)}+\sum_{p \in \mathcal{P}\left(\rightarrow s\left(\alpha_{j}\right)\right)} k_{s\left(\alpha_{j} p\right)}
$$

for

$$
\begin{aligned}
& x_{s\left(\alpha_{j}\right)}+k_{s\left(\alpha_{j}\right)}+\sum_{p \in \mathcal{P}\left(\rightarrow s\left(\alpha_{j}\right)\right)} k_{s(p)} \in \operatorname{rMon}(X)_{s\left(\alpha_{j}\right)} \\
& \quad=X_{s\left(\alpha_{j}\right)} \oplus I K_{s\left(\alpha_{j}\right)} \oplus \bigoplus_{p \in \mathcal{P}\left(\rightarrow s\left(\alpha_{j}\right)\right)} I K_{s(p)} .
\end{aligned}
$$

For $y \in Y_{s\left(\alpha_{j}\right)}$, suppose

$$
h_{s\left(\alpha_{j}\right)}(y)=x_{s\left(\alpha_{j}\right)}+k_{s\left(\alpha_{j}\right)}+\sum_{p \in \mathcal{P}\left(\rightarrow s\left(\alpha_{j}\right)\right)} k_{s(p)} \in \operatorname{rMon}(X)_{s\left(\alpha_{j}\right)} .
$$


Then we have

$$
\begin{aligned}
\operatorname{rMon}(X)_{\alpha_{j}} h_{s\left(\alpha_{j}\right)}(y) & =X_{\alpha_{j}}\left(x_{s\left(\alpha_{j}\right)}\right)+\varphi_{\alpha_{j}}\left(x_{s\left(\alpha_{j}\right)}\right)+k_{s\left(\alpha_{j}\right)}+\sum_{p \in \mathcal{P}\left(\rightarrow s\left(\alpha_{j}\right)\right)} k_{s\left(\alpha_{j} p\right)} \\
& =X_{\alpha_{j}}\left(x_{s\left(\alpha_{j}\right)}\right)+B_{j} h_{s\left(\alpha_{j}\right)}(y) \\
& =X_{\alpha_{j}}\left(f_{s\left(\alpha_{j}\right)} h_{s\left(\alpha_{j}\right)}(y)\right)+B_{j} h_{s\left(\alpha_{j}\right)}(y) \\
& =X_{\alpha_{j}} g_{s\left(\alpha_{j}\right)}(y)+B_{j} h_{s\left(\alpha_{j}\right)}(y) \\
& =g_{1} Y_{\alpha_{j}}(y)+\eta Y_{\alpha_{j}}(y),
\end{aligned}
$$

where the last equality uses the fact that $g: Y \rightarrow X$ is a morphism in $\operatorname{Rep}(Q, A)$.

Now we define $h_{1}: Y_{1} \rightarrow \operatorname{rMon}(X)_{1}$ to be the $A$-map given by

$$
h_{1}(y)=g_{1}(y)+\eta(y)
$$

for each $y \in Y_{1}$. From the computation above we have $\operatorname{rMon}(X)_{\alpha_{j}} h_{s\left(\alpha_{j}\right)}=h_{1} Y_{\alpha_{j}}$ for $j=1, \ldots, m$. It follows that

$$
h=\left(\begin{array}{c}
h_{1} \\
\vdots \\
h_{n}
\end{array}\right): Y \rightarrow \operatorname{rMon}(X)
$$

is a morphism in $\operatorname{Rep}(Q, A)$. Since $f_{1}: \operatorname{rMon}(X)_{1} \rightarrow X_{1}$ is the canonical projection, we have $f_{1} \eta=0$ and $f_{1} g_{1}=g_{1}$, and hence $f h=g$. This completes the proof.

3C. Proof of Theorem 3.1. By Corollary 2.5 and Proposition 3.3 we know that $\operatorname{Mon}(Q, A)$ is a resolving, contravariantly finite subcategory of $\operatorname{Rep}(Q, A)$, and hence $\operatorname{Mon}(Q, A)$ is functorially finite $\operatorname{in} \operatorname{Rep}(Q, A)$; see [Krause and Solberg 2003, Corollary 2.6(i)]. It follows that $\operatorname{Mon}(Q, A)$ has Auslander-Reiten sequences, by [Auslander and Smalø 1981, Theorem 2.4].

\section{Gorenstein-projective modules over the upper triangular matrix algebras}

4A. Let $A$ and $B$ be rings, $M$ an $A$-B-bimodule, and $\Lambda=\left(\begin{array}{cc}A & M \\ 0 & B\end{array}\right)$ the upper triangular matrix ring, where the addition and multiplication are given by the ones of matrices. We assume that $\Lambda$ is an Artin algebra [Auslander et al. 1995, p. 72], and consider finitely generated $\Lambda$-modules. A $\Lambda$-module can be identified with a triple $\left(\begin{array}{l}X \\ Y\end{array}\right)_{\phi}$, or simply $\left(\begin{array}{l}X \\ Y\end{array}\right)$ if $\phi$ is clear, where $X \in A$-mod, $Y \in B$-mod, and $\phi: M \otimes_{B} Y \rightarrow X$ is an $A$-map. A $\Lambda$-map $\left(\begin{array}{l}X \\ Y\end{array}\right)_{\phi} \rightarrow\left(\begin{array}{c}X^{\prime} \\ Y^{\prime}\end{array}\right)_{\phi^{\prime}}$ can be identified with a pair $\left(\begin{array}{l}f \\ g\end{array}\right)$, where $f \in \operatorname{Hom}_{A}\left(X, X^{\prime}\right), g \in \operatorname{Hom}_{B}\left(Y, Y^{\prime}\right)$ are such that the diagram

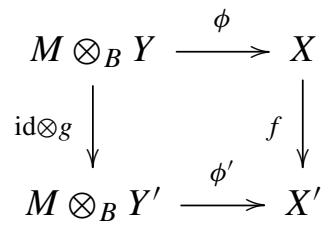


commutes. A sequence of $\Lambda$-maps

$$
0 \rightarrow\left(\begin{array}{c}
X_{1} \\
Y_{1}
\end{array}\right)_{\phi_{1}} \stackrel{\left(\begin{array}{l}
f_{1} \\
g_{1}
\end{array}\right)}{\longrightarrow}\left(\begin{array}{c}
X_{2} \\
Y_{2}
\end{array}\right)_{\phi_{2}} \stackrel{\left(\begin{array}{l}
f_{2} \\
g_{2}
\end{array}\right)}{\longrightarrow}\left(\begin{array}{c}
X_{3} \\
Y_{3}
\end{array}\right)_{\phi_{3}} \rightarrow 0
$$

is exact if and only if

$$
0 \longrightarrow X_{1} \stackrel{f_{1}}{\longrightarrow} X_{2} \stackrel{f_{2}}{\longrightarrow} X_{3} \longrightarrow 0
$$

is an exact sequence of $A$-maps, and

$$
0 \longrightarrow Y_{1} \stackrel{g_{1}}{\longrightarrow} Y_{2} \stackrel{g_{2}}{\longrightarrow} Y_{3} \longrightarrow 0
$$

is an exact sequence of $B$-maps. The indecomposable projective $\Lambda$-modules are exactly

$$
\left(\begin{array}{l}
P \\
0
\end{array}\right) \text { and }\left(\begin{array}{c}
M \otimes_{B} Q \\
Q
\end{array}\right)_{\mathrm{id}},
$$

where $P$ runs over indecomposable projective $A$-modules and $Q$ runs over indecomposable projective $B$-modules.

Note that an algebra $\Lambda$ is of the form above if and only if there is an idempotent decomposition $1=e+f$ such that $f \Lambda e=0$; and in this case

$$
\Lambda=\left(\begin{array}{cc}
e \Lambda e & e \Lambda f \\
0 & f \Lambda f
\end{array}\right)
$$

4B. The following result describes the Gorenstein-projective $\Lambda$-modules, if ${ }_{A} M$ and $M_{B}$ are projective modules.

Theorem 4.1. Let

$$
\Lambda=\left(\begin{array}{cc}
A & M \\
0 & B
\end{array}\right)
$$

be an Artin algebra, $M$ an $A$-B-bimodule such that ${ }_{A} M$ and $M_{B}$ are projective modules. Then

$$
\left(\begin{array}{l}
X \\
Y
\end{array}\right)_{\phi} \in \mathcal{G P}(\Lambda)
$$

if and only if $\phi: M \otimes_{B} Y \rightarrow X$ is injective, Coker $\phi \in \mathcal{G P}(A)$, and $Y \in \mathcal{G P}(B)$. In this case, $X \in \mathcal{G P}(A)$ if and only if $M \otimes_{B} Y \in \mathcal{G P}(A)$.

Note that here $\Lambda$ is not assumed to be Gorenstein: this will be important to the main result in the next section. The same result under the assumption that $\Lambda$ is Gorenstein can be found in [Xiong and Zhang 2012, Corollary 3.3] (however, the proof there cannot be generalized to the non-Gorenstein case). The same corollary implies that, if $\Lambda$ is Gorenstein in Theorem 4.1, $\left(\begin{array}{l}X \\ Y\end{array}\right)_{\phi} \in \mathcal{G P}(\Lambda)$ implies $X \in \mathcal{G P}(A)$. 
Proof of Theorem 4.1. The last assertion is easy: it follows from the exact sequence

$$
0 \longrightarrow M \otimes_{B} Y \stackrel{\phi}{\longrightarrow} X \longrightarrow \text { Coker } \phi \longrightarrow 0
$$

and the fact that $\mathcal{G P}(A)$ is closed under extensions and the kernels of epimorphisms; see, for example, [Holm 2004].

We next prove the "if" part of the first equivalence in the theorem. We assume that $\phi: M \otimes_{B} Y \rightarrow X$ is injective, Coker $\phi \in \mathcal{G P}(A)$, and $Y \in \mathcal{G P}(B)$. Then we have a complete $B$-projective resolution

$$
Q^{\bullet}=\cdots \longrightarrow Q^{-1} \longrightarrow Q^{0} \stackrel{d^{0}}{\longrightarrow} Q^{1} \longrightarrow \cdots
$$

with $Y=\operatorname{Ker} d^{\prime 0}$, and a complete $A$-projective resolution

$$
P^{\bullet}=\cdots \longrightarrow P^{-1} \longrightarrow P^{0} \stackrel{d^{0}}{\longrightarrow} P^{1} \longrightarrow \cdots
$$

with Coker $\phi=\operatorname{Ker} d^{0}$. Since $M_{B}$ is projective, we get the following exact sequences of $A$-modules:

$$
\begin{aligned}
& 0 \rightarrow M \otimes_{B} Y \rightarrow M \otimes_{B} Q^{0} \rightarrow M \otimes_{B} Q^{1} \rightarrow \cdots, \\
& 0 \rightarrow \text { Coker } \phi \rightarrow P^{0} \rightarrow P^{1} \rightarrow \cdots .
\end{aligned}
$$

Since ${ }_{A} M$ is projective, $M \otimes_{B} Q^{i}$ is a projective $A$-module for each $i \geq 0$. Since $\operatorname{Ext}_{A}^{1}\left(\operatorname{Coker} \phi, M \otimes_{B} Q^{0}\right)=0$, it follows from the exact sequence

$$
0 \rightarrow M \otimes_{B} Y \stackrel{\phi}{\rightarrow} X \rightarrow \operatorname{Coker} \phi \rightarrow 0
$$

that the map $M \otimes_{B} Y \rightarrow M \otimes_{B} Q^{0}$ factors through $\phi$. So, by a version of the horseshoe lemma, we see that there is an exact sequence of $A$-modules

$$
0 \rightarrow X \rightarrow P^{0} \oplus\left(M \otimes_{B} Q^{0}\right) \stackrel{\partial^{0}}{\longrightarrow} P^{1} \oplus\left(M \otimes_{B} Q^{1}\right) \rightarrow \cdots
$$

with

$$
\partial^{i}=\left(\begin{array}{cc}
d^{i} & 0 \\
\sigma^{i} & \mathrm{id} \otimes_{B} d^{i}
\end{array}\right), \quad \sigma^{i}: P^{i} \rightarrow M \otimes_{B} Q^{i}
$$

for all $i \in \mathbb{Z}$, such that the diagram

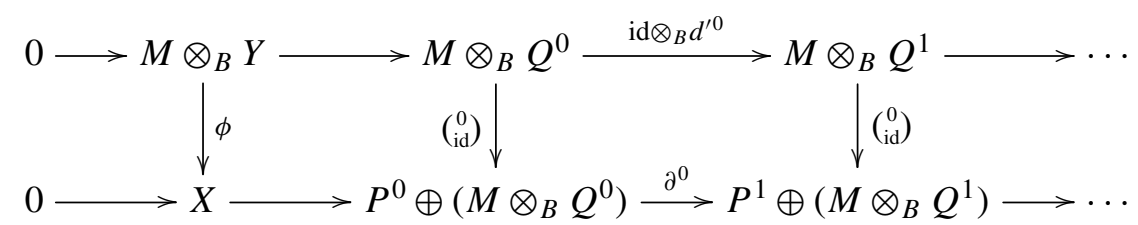


commutes. By the same argument we get the following commutative diagram with exact rows:

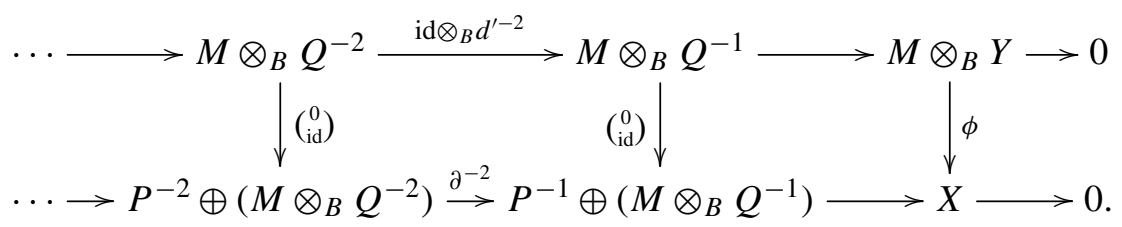

Putting (4-4) and (4-5) together, we get the exact sequence of projective $\Lambda$-modules

$$
\begin{aligned}
L^{\cdot}= & \cdots \\
& \longrightarrow\left(\begin{array}{c}
P^{-1} \oplus\left(M \otimes_{B} Q^{-1}\right) \\
Q^{-1}
\end{array}\right) \\
& \longrightarrow\left(\begin{array}{c}
P^{0} \oplus\left(M \otimes_{B} Q^{0}\right) \\
Q^{0}
\end{array}\right)_{\left(\begin{array}{l}
0 \\
\text { id }
\end{array}\right)} \stackrel{\left(\begin{array}{c}
\partial^{0} \\
d^{\prime 0}
\end{array}\right)}{\longrightarrow}\left(\begin{array}{c}
P^{1} \oplus\left(M \otimes_{B} Q^{1}\right) \\
Q^{1}
\end{array}\right) \longrightarrow \cdots
\end{aligned}
$$

with $\operatorname{Ker}\left(\begin{array}{c}\partial^{0} \\ d^{\prime 0}\end{array}\right)=\left(\begin{array}{c}X \\ Y\end{array}\right)_{\phi}$.

For each projective $A$-module $P, \operatorname{Hom}_{\Lambda}\left(L^{\bullet},\left(\begin{array}{c}P \\ 0\end{array}\right)\right) \cong \operatorname{Hom}_{A}\left(P^{\bullet}, P\right)$ is exact, since $P^{\bullet}$ is a complete projective resolution. For each projective $B$-module $Q$, since $Q^{\bullet}$ is a complete projective resolution, $\operatorname{Hom}_{B}\left(Q^{\bullet}, Q\right)$ is exact. Since $M \otimes_{B} Q$ is projective, $\operatorname{Hom}_{A}\left(P^{\bullet}, M \otimes_{B} Q\right)$ is exact. Note that

$$
\operatorname{Hom}_{\Lambda}\left(L^{\bullet},\left(\begin{array}{c}
M \otimes_{B} Q \\
Q
\end{array}\right)\right) \cong \operatorname{Hom}_{A}\left(P^{\bullet}, M \otimes_{B} Q\right) \oplus \operatorname{Hom}_{B}\left(Q^{\bullet}, Q\right) ;
$$

here the direct sum only means that each term of the complex at the left side is a direct sum of terms of complexes at the right side, that is, it does not mean a direct sum of complexes; in fact, the complex at the right side has differentials

$$
\left(\begin{array}{cc}
\operatorname{Hom}_{A}\left(d^{i}, M \otimes_{B} Q\right) & \operatorname{Hom}_{A}\left(\sigma^{i}, M \otimes_{B} Q\right) \\
0 & \operatorname{Hom}_{B}\left(d^{\prime i}, Q\right)
\end{array}\right) .
$$

By the canonical exact sequence of complexes

$0 \rightarrow \operatorname{Hom}_{A}\left(P^{\bullet}, M \otimes_{B} Q\right) \stackrel{\left(\begin{array}{c}\mathrm{id} \\ 0\end{array}\right)}{\longrightarrow} \operatorname{Hom}_{\Lambda}\left(L^{\bullet},\left(\begin{array}{c}M \otimes_{B} Q \\ Q\end{array}\right)\right) \stackrel{(0 \text { id })}{\longrightarrow} \operatorname{Hom}_{B}\left(Q^{\bullet}, Q\right) \rightarrow 0$,

we know that

$$
\operatorname{Hom}_{\Lambda}\left(L \cdot\left(\begin{array}{c}
M \otimes_{B} Q \\
Q
\end{array}\right)\right)
$$

is also exact. We conclude that $L$ is a complete $\Lambda$-projective resolution, and hence $\left(\begin{array}{l}X \\ Y\end{array}\right)_{\phi}$ is a Gorenstein-projective $\Lambda$-module.

Conversely, assume that $\left(\begin{array}{l}X \\ Y\end{array}\right)_{\phi} \in \mathcal{G} \mathcal{P}(\Lambda)$. Then there is a complete $\Lambda$-projective resolution (4-6) with

$$
\operatorname{Ker}\left(\begin{array}{c}
\partial^{0} \\
d^{\prime 0}
\end{array}\right)=\left(\begin{array}{l}
X \\
Y
\end{array}\right)_{\phi}
$$


Then we get an exact sequence (4-1) of projective $B$-modules with $\operatorname{Ker} d^{\prime 0}=Y$, and the exact sequence

(4-7) $V^{\bullet}=$

$$
\cdots \rightarrow P^{-1} \oplus\left(M \otimes_{B} Q^{-1}\right) \rightarrow P^{0} \oplus\left(M \otimes_{B} Q^{0}\right) \stackrel{\partial^{0}}{\longrightarrow} P^{1} \oplus\left(M \otimes_{B} Q^{1}\right) \rightarrow \cdots
$$

of projective $A$-modules with $\operatorname{Ker} \partial^{0}=X$. Since $M_{B}$ is projective, it follows that $M \otimes_{B} Q^{\bullet}$ is exact. Since $\left(\begin{array}{c}\partial^{i} \\ d^{i}\end{array}\right)$ is a $\Lambda$-map, by (4-6) we know that $\partial^{i}$ is of the form

$$
\partial^{i}=\left(\begin{array}{cc}
d^{i} & 0 \\
\sigma^{i} & \mathrm{id} \otimes_{B} d^{i}
\end{array}\right),
$$

where $\sigma^{i}: P^{i} \rightarrow M \otimes_{B} Q^{i}$ for all $i \in \mathbb{Z}$, and

$$
P^{\bullet}=\cdots \longrightarrow P^{-1} \longrightarrow P^{0} \stackrel{d^{0}}{\longrightarrow} P^{1} \longrightarrow \cdots
$$

is a complex. By the canonical exact sequence of complexes

$$
0 \longrightarrow M \otimes_{B} Q^{\bullet} \stackrel{\left(\begin{array}{c}
\text { id } \\
0
\end{array}\right)}{\longrightarrow} V^{\bullet} \stackrel{(0 \text { id })}{\longrightarrow} \operatorname{Hom}_{B}\left(Q^{\bullet}, Q\right) P^{\bullet} \longrightarrow 0,
$$

we see that $P^{\bullet}$ is also exact.

From (4-6) we have the following commutative diagram with exact rows and columns:

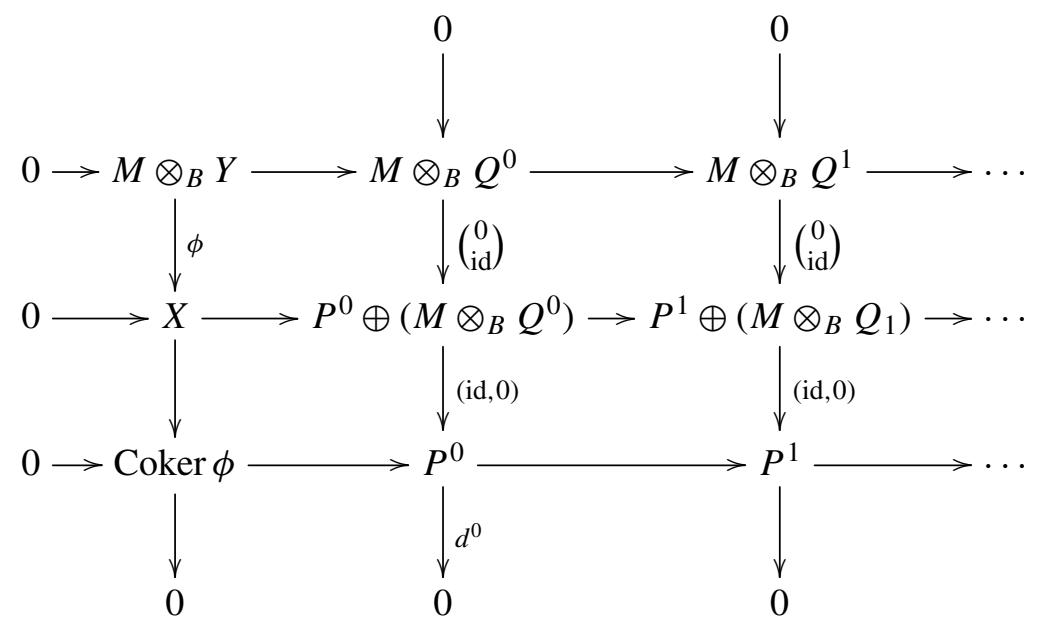

Thus $\phi: M \otimes_{B} Y \longrightarrow X$ is injective and $\operatorname{Ker} d^{0} \cong$ Coker $\phi$. For each projective $A$-module $P$, since

$$
\operatorname{Hom}_{\Lambda}\left(L^{\bullet},\left(\begin{array}{c}
P \\
0
\end{array}\right)\right) \cong \operatorname{Hom}_{A}\left(P^{\bullet}, P\right)
$$

and $L^{\bullet}$ is a complete projective resolution, it follows that $P^{\bullet}$ is a complete projective resolution, and hence Coker $\phi$ is a Gorenstein-projective $A$-module. 
For each projective $B$-module $Q$, since $P$ is a complete projective resolution, it follows that $\operatorname{Hom}_{A}\left(P^{\bullet}, M \otimes_{B} Q\right)$ is exact. Since $L^{\bullet}$ is a complete projective resolution, it follows that

$$
\operatorname{Hom}_{\Lambda}\left(L^{\bullet},\left(\begin{array}{c}
M \otimes_{B} Q \\
Q
\end{array}\right)\right) \cong \operatorname{Hom}_{A}\left(P^{\bullet}, M \otimes_{B} Q\right) \oplus \operatorname{Hom}_{B}\left(Q^{\bullet}, Q\right)
$$

is exact (again, the direct sum does not mean a direct sum of complexes). By the same argument we know that $\operatorname{Hom}_{B}\left(Q^{\bullet}, Q\right)$ is exact. It follows that $Y$ is a Gorenstein-projective $B$-module.

\section{Main result}

5A. The aim of this section is to prove the following characterization of Gorensteinprojective $\Lambda$-modules, where $\Lambda$ is the path algebra of a finite acyclic quiver over a finite-dimensional algebra. We emphasize that here $\Lambda$ is not assumed to be Gorenstein.

Theorem 5.1. Let $Q$ be a finite acyclic quiver, and $A$ a finite-dimensional algebra over a field $k$. Let $\Lambda=A \otimes_{k} k Q$, and $X=\left(X_{i}, X_{\alpha}, i \in Q_{0}, \alpha \in Q_{1}\right)$ be a $\Lambda$-module. Then $X \in \mathcal{G P}(\Lambda)$ if and only if $X \in \operatorname{Mon}(Q, A)$ and $X$ satisfies this condition:

(G) for each $i \in Q_{0}, X_{i}$ and the quotient $X_{i} / \underset{\substack{\alpha \in Q_{1} \\ e(\alpha)=i}}{\bigoplus} \operatorname{Im} X_{\alpha}$ lie in $\mathcal{G P}(A)$.

Example 5.2. (i) Taking

$$
Q=\bullet \rightarrow \cdots \rightarrow \bullet_{1}
$$

in Theorem 5.1, we get that a $T_{n}(A)$-module $X=\left(X_{i}, \phi_{i}\right)$ is Gorenstein-projective if and only if each $\phi_{i}$ is injective and that each $X_{i}$ is a Gorenstein-projective $A$-module and each Coker $\phi_{i}$ is a Gorenstein-projective $A$-module. Under the assumption that $A$ is Gorenstein, this result has been obtained in [Zhang 2011, Corollary 4.1]; the case for $n=2$ was treated in [Li and Zhang 2010, Theorem 1.1(i)]; see also [Iyama et al. 2011, Proposition 3.6(i)].

(ii) Let $\Lambda$ be the $k$-algebra given by quiver

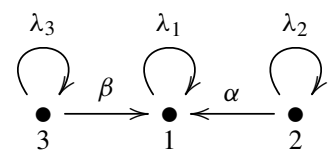

with relations $\lambda_{1}^{2}, \lambda_{2}^{2}, \lambda_{3}^{2}, \alpha \lambda_{2}-\lambda_{1} \alpha, \beta \lambda_{3}-\lambda_{1} \beta$. Then

$$
\Lambda=A \otimes_{k} k Q=\left(\begin{array}{ccc}
A & A & A \\
0 & A & 0 \\
0 & 0 & A
\end{array}\right)
$$


where $Q$ is the quiver

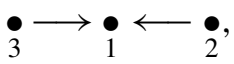

and $A=k[x] /\left\langle x^{2}\right\rangle$. Let $k$ be the simple $A$-module, and $\sigma: k \hookrightarrow A$ the inclusion. By Theorem 5.1, the following $\Lambda$-modules lie in $\operatorname{GP}(\Lambda)$ :

$$
\begin{aligned}
& \left(X_{1}=A, X_{2}=0, X_{3}=0, \quad X_{\alpha}=0=X_{\beta}\right), \\
& \left(X_{1}=A, X_{2}=A, X_{3}=0, \quad X_{\alpha}=\mathrm{id}, X_{\beta}=0\right), \\
& \left(X_{1}=A, X_{2}=0, X_{3}=A, X_{\alpha}=0, X_{\beta}=\mathrm{id}\right), \\
& \left(X_{1}=k, X_{2}=0, X_{3}=0, X_{\alpha}=0=X_{\beta}\right), \\
& \left(X_{1}=k, X_{2}=k, X_{3}=0, \quad X_{\alpha}=\mathrm{id}, X_{\beta}=0\right), \\
& \left(X_{1}=k, X_{2}=0, X_{3}=k, X_{\alpha}=0, X_{\beta}=\mathrm{id}\right), \\
& \left(X_{1}=A, X_{2}=k, X_{3}=0, X_{\alpha}=\sigma, X_{\beta}=0\right), \\
& \left(X_{1}=A, X_{2}=0, X_{3}=k, X_{\alpha}=0, X_{\beta}=\sigma\right), \\
& \left(X_{1}=A \oplus k, X_{2}=k, X_{3}=k, X_{\alpha}=\left(\begin{array}{c}
0 \\
\mathrm{id}
\end{array}\right), X_{\beta}=\left(\begin{array}{c}
\sigma \\
\mathrm{id}
\end{array}\right)\right) .
\end{aligned}
$$

In fact this is the complete list of the pairwise nonisomorphic indecomposable Gorenstein-projective $\Lambda$-modules. Also by Theorem 5.1,

$$
\left(Y_{1}=A, Y_{2}=k, Y_{3}=k, Y_{\alpha}=\sigma=Y_{\beta}\right) \notin \mathcal{G P}(\Lambda) .
$$

For a description of all the pairwise nonisomorphic indecomposable Gorensteinprojective $\Lambda$-modules see [Ringel and Zhang 2011], where $\Lambda$ is the path algebra of an arbitrary acyclic quiver over $A=k[x] /\left\langle x^{2}\right\rangle$.

5B. We prove Theorem 5.1 by using Theorem 4.1 and induction on $\left|Q_{0}\right|$.

Remember we label $Q_{0}$ as $1, \ldots, n$, in such a way that $j>i$ if $\alpha: j \rightarrow i$ is in $Q_{1}$. Thus $n$ is a source of $Q$. Denote by $Q^{\prime}$ the quiver obtained from $Q$ by deleting vertex $n$, and $\Lambda^{\prime}=A \otimes_{k} k Q^{\prime}$. Let $P(n)$ be the indecomposable projective (left) $k Q$-module at vertex $n$. Put $P=A \otimes_{k} \operatorname{rad} P(n)$. Clearly $P$ is a $\Lambda^{\prime}$ - $A$-bimodule and $\Lambda=\left(\begin{array}{cc}\Lambda^{\prime} & P \\ 0 & A\end{array}\right)$; compare $(2-2)$.

Since $k Q$ is hereditary, $\operatorname{rad} P(n)$ is a projective $k Q^{\prime}$-module, and hence $P=$ $A \otimes_{k} \operatorname{rad} P(n)$ is a (left) projective $\Lambda^{\prime}$-module, and a (right) projective $A$-module (since as a right $A$-module, $P$ is a direct sum of copies of $A_{A}$ ). So we can apply Theorem 4.1. For this, we write a $\Lambda$-module $X=\left(X_{i}, X_{\alpha}, i \in Q_{0}, \alpha \in Q_{1}\right)$ as $X=\left(\begin{array}{l}X^{\prime} \\ X_{n}\end{array}\right)_{\phi}$, where $X^{\prime}=\left(X_{i}, X_{\alpha}, i \in Q_{0}^{\prime}, \alpha \in Q_{1}^{\prime}\right)$ is a $\Lambda^{\prime}$-module, and

$$
\phi: P \otimes_{A} X_{n} \rightarrow X^{\prime}
$$

is a $\Lambda^{\prime}$-map. The explicit expression of $\phi$ is given in the proof of Lemma 5.4. We keep all these notations of $Q^{\prime}, \Lambda^{\prime}, P(n), P, X^{\prime}$ and $\phi$ throughout this section. 
5C. By a direct translation from Theorem 4.1 in this special case, we have:

Lemma 5.3. Let $X=\left(\begin{array}{c}X^{\prime} \\ X_{n}\end{array}\right)_{\phi}$ be a $\Lambda$-module. Then $X \in \mathcal{G P}(\Lambda)$ if and only if $X$ satisfies the following conditions:

(i) $X_{n} \in \mathcal{G P}(A)$.

(ii) $\phi: P \otimes_{A} X_{n} \rightarrow X^{\prime}$ is injective.

(iii) $\operatorname{Coker} \phi \in \mathcal{G P}\left(\Lambda^{\prime}\right)$.

For each $i \in Q_{0}^{\prime}$, put $\mathcal{A}(n \rightarrow i)$ to be the set of arrows from $n$ to $i$; and $\mathcal{P}(n \rightarrow i)$ the set of paths from $n$ to $i$. For an integer $m \geq 0$ and a module $M$, let $M^{m}$ denote the direct sum of $m$ copies of $M$.

Lemma 5.4. Let $X=\left(X_{i}, X_{\alpha}, i \in Q_{0}, \alpha \in Q_{1}\right)$ be a $\Lambda$-module. If $X_{\beta}$ is injective for each $\beta \in Q_{1}^{\prime}, \phi: P \otimes_{A} X_{n} \rightarrow X^{\prime}$ is injective if and only if $X_{\alpha}$ is injective for all $\alpha \in Q_{1}$, and $\sum_{p \in \mathcal{P}(n \rightarrow i)} \operatorname{Im} X_{p}=\bigoplus_{p \in \mathcal{P}(n \rightarrow i)} \operatorname{Im} X_{p}$ for all $Q_{0}^{\prime}$.

Proof. For $i \in Q_{0}^{\prime}$, set $m_{i}=|\mathcal{P}(n \rightarrow i)|$. As a $k Q^{\prime}$-module, $\operatorname{rad} P(n)$ can be written as

$$
\left(\begin{array}{c}
k^{m_{1}} \\
\vdots \\
k^{m_{n-1}}
\end{array}\right)
$$

(see (2-1) and Section 5B), hence we have isomorphisms of $\Lambda^{\prime}$-modules

$$
P \otimes_{A} X_{n} \cong\left(\operatorname{rad} P(n) \otimes_{k} A\right) \otimes_{A} X_{n} \cong \operatorname{rad} P(n) \otimes_{k} X_{n} \cong\left(\begin{array}{c}
X_{n}^{m_{1}} \\
\vdots \\
X_{n}^{m_{n-1}}
\end{array}\right) \text {. }
$$

Let $\mathcal{P}(n \rightarrow i)=\left\{p_{1}, \ldots, p_{m_{i}}\right\}$. Then $\phi$ is of the form

$$
\left(\begin{array}{c}
\phi_{1} \\
\vdots \\
\phi_{n-1}
\end{array}\right): P \otimes_{A} X_{n} \cong\left(\begin{array}{c}
X_{n}^{m_{1}} \\
\vdots \\
X_{n}^{m_{n-1}}
\end{array}\right) \rightarrow\left(\begin{array}{c}
X_{1} \\
\vdots \\
X_{n-1}
\end{array}\right)
$$

where $\phi_{i}=\left(X_{p_{1}}, \ldots, X_{p_{m_{i}}}\right): X_{n}^{m_{i}} \rightarrow X_{i}$ (for the meaning of $X_{p}$ see Section 2C). So $\phi$ is injective if and only if $\phi_{i}$ is injective for each $i \in Q_{0}^{\prime}$, and if and only if

$$
\sum_{p \in \mathcal{P}(n \rightarrow i)} \operatorname{Im} X_{p}=\bigoplus_{p \in \mathcal{P}(n \rightarrow i)} \operatorname{Im} X_{p} \quad \text { and } \quad X_{p} \text { is injective for all } p \in \mathcal{P}(n \rightarrow i) .
$$

From this and the assumption the assertion follows.

Lemma 5.5. Let $X=\left(\begin{array}{l}X^{\prime} \\ X_{n}\end{array}\right)_{\phi}$ be a monic $\Lambda$-module.

(1) For each $i \in Q_{0}^{\prime}$ there holds $\sum_{p \in \mathcal{P}(n \rightarrow i)} \operatorname{Im} X_{p}=\bigoplus_{p \in \mathcal{P}(n \rightarrow i)} \operatorname{Im} X_{p}$.

(2) $\phi: P \otimes_{A} X_{n} \rightarrow X^{\prime}$ is injective. 
(3) $\operatorname{Coker} \phi=\left(X_{i} / \bigoplus_{p \in \mathcal{P}(n \rightarrow i)} \operatorname{Im} X_{p}, \widetilde{X}_{\alpha}, i \in Q_{0}^{\prime}, \alpha \in Q_{1}^{\prime}\right)$, where, for each $\alpha: j \rightarrow i$ in $Q_{1}^{\prime}$,

$$
\tilde{X}_{\alpha}: X_{j} / \bigoplus_{q \in \mathcal{P}(n \rightarrow j)} \operatorname{Im} X_{q} \rightarrow X_{i} / \bigoplus_{p \in \mathcal{P}(n \rightarrow i)} \operatorname{Im} X_{p}
$$

is the A-map induced by $X_{\alpha}$.

Proof. By Lemma 5.4 and its proof, it suffices to prove (1). For each $i \in Q_{0}^{\prime}$, set $l_{i}=0$ if $\mathcal{P}(n \rightarrow i)$ is empty, and $l_{i}=\max \{l(p) \mid p \in \mathcal{P}(n \rightarrow i)\}$ otherwise, where $l(p)$ is the length of $p$. We use induction on $l_{i}$. If $l_{i}=0$, (1) trivially holds. Suppose $l_{i} \geqslant 1$. Let $\sum_{p \in \mathcal{P}(n \rightarrow i)} X_{p}\left(x_{n, p}\right)=0$ for $x_{n, p} \in X_{n}$. Since

$$
\sum_{p \in \mathcal{P}(n \rightarrow i)-\mathcal{A}(n \rightarrow i)} \operatorname{Im} X_{p}=\sum_{\substack{\alpha \in Q_{1}^{\prime} \\ e(\alpha)=i}} X_{\alpha}\left(\sum_{q \in \mathcal{P}(n \rightarrow s(\alpha))} \operatorname{Im} X_{q}\right),
$$

we have

$$
\begin{aligned}
0=\sum_{p \in \mathcal{P}(n \rightarrow i)} X_{p}\left(x_{n, p}\right) & =\sum_{\alpha \in \mathcal{A}(n \rightarrow i)} X_{\alpha}\left(x_{n, \alpha}\right)+\sum_{p \in \mathcal{P}(n \rightarrow i)-\mathcal{A}(n \rightarrow i)} X_{p}\left(x_{n, p}\right) \\
& =\sum_{\alpha \in \mathcal{A}(n \rightarrow i)} X_{\alpha}\left(x_{n, \alpha}\right)+\sum_{\substack{\beta \in Q_{1}^{\prime} \\
e(\beta)=i}} X_{\beta}\left(\sum_{q \in \mathcal{P}(n \rightarrow s(\beta))} X_{q}\left(x_{n, \beta q}\right)\right) .
\end{aligned}
$$

By (m2) in Definition 2.2 we know that $X_{\alpha}\left(x_{n, \alpha}\right)=0$ for $\alpha \in \mathcal{A}(n \rightarrow i)$, and

$$
X_{\beta}\left(\sum_{q \in \mathcal{P}(n \rightarrow s(\beta))} X_{q}\left(x_{n, \beta q}\right)\right)=0
$$

for $\beta \in Q_{1}^{\prime}$ with $e(\beta)=i$. So $\sum_{q \in \mathcal{P}(n \rightarrow s(\beta))} X_{q}\left(x_{n, \beta q}\right)=0$ by condition (m1) in Definition 2.2. Since $l_{s(\beta)}<l_{i}$ for each $\beta \in Q_{1}^{\prime}$ with $e(\beta)=i$, it follows from the inductive hypothesis that $X_{q}\left(x_{n, \beta q}\right)=0$ for $\beta \in Q_{1}^{\prime}, e(\beta)=i$, and $q \in \mathcal{P}(n \rightarrow s(\beta))$. This proves (1) and the lemma.

Lemma 5.6. Let $X=\left(\begin{array}{l}X^{\prime} \\ X_{n}\end{array}\right)_{\phi}$ be a monic $\Lambda$-module. Then Coker $\phi$ is a monic $\Lambda^{\prime}$-module.

Proof. We need to prove that, for each $i \in Q_{0}^{\prime}$, the $\Lambda^{\prime}$-map

$$
\left(\tilde{X}_{\alpha}\right)_{\substack{\alpha \in Q_{1}^{\prime} \\ e(\alpha)=i}}: \bigoplus_{\substack{\alpha \in Q_{1}^{\prime} \\ e(\alpha)=i}}\left(X_{s(\alpha)} / \bigoplus_{q \in \mathcal{P}(n \rightarrow s(\alpha))} \operatorname{Im} X_{q}\right) \rightarrow X_{i} / \bigoplus_{p \in \mathcal{P}(n \rightarrow i)} \operatorname{Im} X_{p}
$$

is injective. For this, assume that

$$
\sum_{\substack{\alpha \in Q_{1}^{\prime} \\ e(\alpha)=i}} \widetilde{X}_{\alpha}\left(\overline{x_{s(\alpha), \alpha}}\right)=0
$$


where $\overline{x_{s(\alpha), \alpha}}$ is the image of $x_{s(\alpha), \alpha} \in X_{s(\alpha)}$ in $X_{s(\alpha)} / \bigoplus_{q \in \mathcal{P}(n \rightarrow s(\alpha))} \operatorname{Im} X_{q}$. Then

$$
\sum_{\substack{\alpha \in Q_{1}^{\prime} \\ e(\alpha)=i}} X_{\alpha}\left(x_{s(\alpha), \alpha}\right) \in \bigoplus_{p \in \mathcal{P}(n \rightarrow i)} \operatorname{Im} X_{p} .
$$

So there are $x_{n, p} \in X_{n}$ such that

$$
\sum_{\substack{\alpha \in Q_{1}^{\prime} \\ e(\alpha)=i}} X_{\alpha}\left(x_{s(\alpha), \alpha}\right)=\sum_{p \in \mathcal{P}(n \rightarrow i)} X_{p}\left(x_{n, p}\right) .
$$

Thus

$$
\begin{aligned}
0 & =\sum_{\substack{\alpha \in Q_{1}^{\prime} \\
e(\alpha)=i}} X_{\alpha}\left(x_{s(\alpha), \alpha}\right)-\sum_{p \in \mathcal{P}(n \rightarrow i)} X_{p}\left(x_{n, p}\right) \\
& =\sum_{\substack{\alpha \in Q_{1}^{\prime} \\
e(\alpha)=i}} X_{\alpha}\left(x_{s(\alpha), \alpha}\right)-\sum_{\beta \in \mathcal{A}(n \rightarrow i)} X_{\beta}\left(x_{n, \beta}\right)-\sum_{\substack{\alpha \in Q_{1}^{\prime} \\
e(\alpha)=i}} X_{\alpha}\left(\sum_{q \in \mathcal{P}(n \rightarrow s(\alpha))} X_{q}\left(x_{n, \alpha q}\right)\right) \\
& =\sum_{\substack{\alpha \in Q_{1}^{\prime} \\
e(\alpha)=i}} X_{\alpha}\left(x_{s(\alpha), \alpha}-\sum_{q \in \mathcal{P}(n \rightarrow s(\alpha))} X_{q}\left(x_{n, \alpha q}\right)\right)-\sum_{\beta \in \mathcal{A}(n \rightarrow i)} X_{\beta}\left(x_{n, \beta}\right) .
\end{aligned}
$$

Using the assumption on $X$, we get

$$
x_{s(\alpha), \alpha}=\sum_{q \in \mathcal{P}(n \rightarrow s(\alpha))} X_{q}\left(x_{n, \alpha q}\right),
$$

that is, $\overline{x_{s(\alpha), \alpha}}=0$.

Lemma 5.7. Let $X=\left(\begin{array}{c}X^{\prime} \\ X_{n}\end{array}\right)_{\phi}$ be a monic $\Lambda$-module satisfying $(\mathrm{G})$. Then

$$
\left(X_{i} / \bigoplus_{p \in \mathcal{P}(n \rightarrow i)} \operatorname{Im} X_{p}\right) /\left(\bigoplus_{\substack{\alpha \in Q_{1}^{\prime} \\ e(\alpha)=i}} \operatorname{Im} \tilde{X}_{\alpha}\right)
$$

is a Gorenstein-projective A-module for all $i \in Q_{0}^{\prime}$.

Proof. Since

$$
\bigoplus_{p \in \mathcal{P}(n \rightarrow i)-\mathcal{A}(n \rightarrow i)} \operatorname{Im} X_{p} \subseteq \sum_{\substack{\beta \in Q_{1} \\ e(\beta)=i}} \operatorname{Im} X_{\beta}
$$

it follows that

$$
\sum_{\substack{\alpha \in Q_{1}^{\prime} \\ e(\alpha)=i}} \operatorname{Im} \tilde{X}_{\alpha}=\left(\sum_{\substack{\alpha \in Q_{1}^{\prime} \\ e(\alpha)=i}} \operatorname{Im} X_{\alpha}+\bigoplus_{p \in \mathcal{P}(n \rightarrow i)} \operatorname{Im} X_{p}\right) /\left(\bigoplus_{p \in \mathcal{P}(n \rightarrow i)} \operatorname{Im} X_{p}\right)
$$




$$
\begin{aligned}
& =\left(\sum_{\substack{\beta \in Q_{1} \\
e(\beta)=i}} \operatorname{Im} X_{\beta}+\bigoplus_{p \in \mathcal{P}(n \rightarrow i)-\mathcal{A}(n \rightarrow i)} \operatorname{Im} X_{p}\right) /\left(\bigoplus_{p \in \mathcal{P}(n \rightarrow i)} \operatorname{Im} X_{p}\right) \\
& =\left(\sum_{\substack{\beta \in Q_{1} \\
e(\beta)=i}} \operatorname{Im} X_{\beta}\right) /\left(\bigoplus_{p \in \mathcal{P}(n \rightarrow i)} \operatorname{Im} X_{p}\right) \\
& =\left(\underset{\substack{\beta \in Q_{1} \\
e(\beta)=i}}{\bigoplus} \operatorname{Im} X_{\beta}\right) /\left(\bigoplus_{p \in \mathcal{P}(n \rightarrow i)} \operatorname{Im} X_{p}\right)
\end{aligned}
$$

(the last equality following by $(\mathrm{m} 2)$ in Definition 2.2). Hence the desired quotient is $X_{i} / \bigoplus_{\beta \in Q_{1}} \operatorname{Im} X_{\beta}$, which is Gorenstein-projective by $(\mathrm{G})$.

Lemma 5.8. Let $X=\left(\begin{array}{c}X^{\prime} \\ X_{n}\end{array}\right)_{\phi}$ be a monic $\Lambda$-module satisfying $(\mathrm{G})$. Then

$$
X_{i} / \bigoplus_{p \in \mathcal{P}(n \rightarrow j)} \operatorname{Im} X_{p}
$$

is a Gorenstein-projective A-module for each $i \in Q_{0}^{\prime}$.

Proof. We prove the assertion by using induction on $l_{i}$, which is defined in the proof of Lemma 5.5. If $i \in Q_{0}^{\prime}$ with $l_{i}=0$, the assertion follows from $(\mathrm{G})$.

Suppose $l_{i} \geqslant 1$. Since $\bigoplus_{p \in \mathcal{P}(n \rightarrow i)} \operatorname{Im} X_{p} \subseteq \bigoplus_{\substack{\alpha \in Q_{1} \\ e(\alpha)=i}} \operatorname{Im} X_{\alpha}$, we have the exact sequence

$$
\begin{aligned}
0 \longrightarrow\left(\bigoplus_{\substack{\alpha \in Q_{1} \\
e(\alpha)=i}} \operatorname{Im} X_{\alpha}\right) /\left(\bigoplus_{p \in \mathcal{P}(n \rightarrow i)} \operatorname{Im} X_{p}\right) & \\
& \longrightarrow X_{i} / \bigoplus_{p \in \mathcal{P}(n \rightarrow i)} \operatorname{Im} X_{p} \longrightarrow X_{i} / \bigoplus_{\substack{\alpha \in Q_{1} \\
e(\alpha)=i}} \operatorname{Im} X_{\alpha} \longrightarrow 0,
\end{aligned}
$$

and by $(\mathrm{G})$ the last term on the second line is Gorenstein-projective. It suffices to prove that the term on the first line is Gorenstein-projective. By (5-1) this term is $\bigoplus_{\alpha \in Q_{1}^{\prime}} \operatorname{Im} \widetilde{X}_{\alpha}$. By Lemma 5.6 each $\widetilde{X}_{\alpha}$ is injective, and it follows that $e(\alpha)=i$

$$
\operatorname{Im} \widetilde{X}_{\alpha} \cong X_{j} / \bigoplus_{p \in \mathcal{P}(n \rightarrow j)} \operatorname{Im} X_{p},
$$

where $j=s(\alpha)$. Since $l_{j}<l_{i}$, the conclusion of the lemma follows from the inductive hypothesis.

Lemma 5.9. The sufficiency in Theorem 5.1 holds. That is, if

$$
X=\left(X_{i}, X_{\alpha}, i \in Q_{0}, \alpha \in Q_{1}\right)
$$

is a monic $\Lambda$-module satisfying $(\mathrm{G}), X$ is Gorenstein-projective. 
Proof. Using induction on $n=\left|Q_{0}\right|$, the assertion clearly holds for $n=1$. Suppose that the assertion holds for $n-1$ with $n \geq 2$. It suffices to prove that $X$ satisfies Lemma 5.3(i)-(iii).

Condition (i) is contained in (G); and condition (ii) follows from Lemma 5.5(2). By Lemma 5.6 Coker $\phi$ is a monic $\Lambda^{\prime}$-module; and by Lemmas 5.7 and 5.8 we know that Coker $\phi$ satisfies (G). It follows from the inductive hypothesis that condition (iii) is satisfied.

Lemma 5.10. Let $X=\left(X_{i}, X_{\alpha}, i \in Q_{0}, \alpha \in Q_{1}\right)$ be a $\Lambda$-module with $X_{n}$ a Gorenstein-projective A-module. Then $P \otimes_{A} X_{n}$ is a Gorenstein-projective $\Lambda^{\prime}$ module, where $P$ is defined in Section $5 B$.

Proof. Let $P(n)$ be the indecomposable projective $k Q$-module at vertex $n$. Writing $\operatorname{rad} P(n)$ as a representation of $Q^{\prime}$ over $k$, we have

$$
\operatorname{rad} P(n)=\left(k^{m_{i}}, f_{\alpha}, i \in Q_{0}^{\prime}, \alpha \in Q_{1}^{\prime}\right),
$$

where $m_{i}=|\mathcal{P}(n \rightarrow i)|$ for each $i \in Q_{0}^{\prime}$. By the construction of $P(n)$ we know that $\operatorname{rad} P(n)$ has the following three properties:

(1) Each $f_{\alpha}: k^{m_{s(\alpha)}} \rightarrow k^{m_{e(\alpha)}}$ is injective.

(2) For each $i \in Q_{0}^{\prime}$,

$$
\sum_{\substack{\alpha \in Q_{1}^{\prime} \\ e(\alpha)=i}} \operatorname{Im} f_{\alpha}=\bigoplus_{\substack{\alpha \in Q_{1}^{\prime} \\ e(\alpha)=i}} \operatorname{Im} f_{\alpha} .
$$

(3) For each $i \in Q_{0}^{\prime}, k^{m_{i}} /\left(\bigoplus_{\alpha \in Q_{1}^{\prime}} \operatorname{Im} f_{\alpha}\right)$ and $k^{|\mathcal{A}(n \rightarrow i)|}$ are isomorphic as $k$ spaces.

It follows that

$$
\begin{aligned}
P \otimes_{A} X_{n} & \cong\left(\operatorname{rad} P(n) \otimes_{k} A\right) \otimes_{A} X_{n} \\
& \cong \operatorname{rad} P(n) \otimes_{k} X_{n}=\left(X_{n}^{m_{i}}, f_{\alpha} \otimes_{k} \operatorname{id}_{X_{n}}, i \in Q_{0}^{\prime}, \alpha \in Q_{1}^{\prime}\right) .
\end{aligned}
$$

By (1), (2), and (3) we clearly see that $P \otimes_{A} X_{n}$ is a monic $\Lambda^{\prime}$-module satisfying (G); for example, by (3) we know that

$$
X_{n}^{m_{i}} / \bigoplus_{\substack{\alpha \in Q_{1}^{\prime} \\ e(\alpha)=i}} \operatorname{Im}\left(f_{\alpha} \otimes_{k} \mathrm{id}_{X_{n}}\right) \cong X_{n}^{|\mathcal{A}(n \rightarrow i)|}
$$

is a Gorenstein-projective $A$-module. Now the result follows from Lemma 5.9. 
5D. Proof of Theorem 5.1. By Lemma 5.9 it remains to prove necessity, namely, if $X$ is a Gorenstein-projective $\Lambda$-module, $X$ is a monic $\Lambda$-module satisfying (G). Using induction on $n=\left|Q_{0}\right|$, the assertion is clear for $n=1$. Suppose that the assertion holds for $n-1$ with $n \geq 2$. We write $X$ as $\left(\begin{array}{l}X^{\prime} \\ X_{n}\end{array}\right)_{\phi}$. Then $X$ satisfies conclusions (i)-(iii) of Lemma 5.3.

By (i) and Lemma 5.10 we know that $P \otimes_{A} X_{n}$ is a Gorenstein-projective $\Lambda^{\prime}$ module. Then, by (ii) and (iii), we know that $X^{\prime} \in \mathcal{G P}\left(\Lambda^{\prime}\right)$, since $\mathcal{G P}\left(\Lambda^{\prime}\right)$ is closed under extensions. By the inductive hypothesis $X^{\prime}$ is a monic $\Lambda^{\prime}$-module satisfying $(G)$. Hence:

(1) $X_{\beta}$ is injective for each $\beta \in Q_{1}^{\prime}$.

(2) $X_{i}$ is Gorenstein-projective for each $i \in Q_{0}^{\prime}$.

(3) $X_{\alpha}$ is injective for each $\alpha \in Q_{1}$.

(4) $\sum_{p \in \mathcal{P}(n \rightarrow i)} \operatorname{Im} X_{p}=\bigoplus_{p \in \mathcal{P}(n \rightarrow i)} \operatorname{Im} X_{p}$ for all $i \in Q_{0}^{\prime}$.

We get (3) and (4) from (1), condition (ii), and Lemma 5.4.

Since Coker $\phi=\left(X_{i} / \bigoplus_{p \in \mathcal{P}(n \rightarrow i)} \operatorname{Im} X_{p}, \widetilde{X}_{\alpha}, i \in Q_{0}^{\prime}, \alpha \in Q_{1}^{\prime}\right)$ is a Gorensteinprojective $\Lambda^{\prime}$-module, it follows from the inductive hypothesis that the following properties hold:

(5) For each $\alpha \in Q_{1}^{\prime}, \widetilde{X}_{\alpha}$ is injective.

(6) $\sum_{\substack{\alpha \in Q_{1}^{\prime} \\ e(\alpha)=i}} \operatorname{Im} \widetilde{X}_{\alpha}=\bigoplus_{\substack{\alpha \in Q_{1}^{\prime} \\ e(\alpha)=i}} \operatorname{Im} \widetilde{X}_{\alpha}$, for all $i \in Q_{0}^{\prime}$.

Claim 1: X satisfies (m2) in Definition 2.2.

Indeed, suppose

$$
\sum_{\substack{\alpha \in Q_{1} \\ e(\alpha)=i}} X_{\alpha}\left(x_{s(\alpha), \alpha}\right)=0
$$

Since

$$
\sum_{\substack{\alpha \in Q_{1} \\ e(\alpha)=i}} X_{\alpha}\left(x_{s(\alpha), \alpha}\right)=\sum_{\alpha \in \mathcal{A}(n \rightarrow i)} X_{\alpha}\left(x_{s(\alpha), \alpha}\right)+\sum_{\substack{\alpha \in Q_{1}^{\prime} \\ e(\alpha)=i}} X_{\alpha}\left(x_{s(\alpha), \alpha}\right),
$$

it follows that

$$
\begin{aligned}
\sum_{\substack{\alpha \in Q_{1}^{\prime} \\
e(\alpha)=i}} \tilde{X}_{\alpha}\left(\overline{x_{s(\alpha), \alpha}}\right) & =\sum_{\substack{\alpha \in Q_{1}^{\prime} \\
e(\alpha)=i}} X_{\alpha}\left(x_{s(\alpha), \alpha}\right)+\bigoplus_{p \in \mathcal{P}(n \rightarrow i)} \operatorname{Im} X_{p} \\
& =-\sum_{\alpha \in \mathcal{A}(n \rightarrow i)} X_{\alpha}\left(x_{s(\alpha), \alpha}\right)+\bigoplus_{p \in \mathcal{P}(n \rightarrow i)} \operatorname{Im} X_{p}=0,
\end{aligned}
$$

where we used (5-2) for the second equality. 
Then by (6) we have $\widetilde{X}_{\alpha}\left(\overline{x_{s(\alpha), \alpha}}\right)=0$; and by (5) we know $\overline{x_{s(\alpha), \alpha}}=0$ for each $\alpha \in Q_{1}^{\prime}$ with $e(\alpha)=i$. This means that there are $x_{n, q} \in X_{n}$ such that

$$
x_{s(\alpha), \alpha}=\sum_{q \in \mathcal{P}(n \rightarrow s(\alpha))} X_{q}\left(x_{n, q}\right) \in \sum_{q \in \mathcal{P}(n \rightarrow s(\alpha))} \operatorname{Im} X_{q}
$$

for each $\alpha \in Q_{1}^{\prime}$ with $e(\alpha)=i$. By (5-2) we have

$$
0=\sum_{\alpha \in \mathcal{A}(n \rightarrow i)} X_{\alpha}\left(x_{n, \alpha}\right)+\sum_{\substack{\alpha \in Q_{1}^{\prime} \\ e(\alpha)=i}} X_{\alpha}\left(\sum_{q \in \mathcal{P}(n \rightarrow s(\alpha))} X_{q}\left(x_{n, q}\right)\right) .
$$

By (4) we know that $X_{\alpha}\left(x_{n, \alpha}\right)=0$ for all $\alpha \in \mathcal{A}(n \rightarrow i)$, and that $X_{\alpha} X_{q}\left(x_{n, q}\right)=0$ for all $\alpha \in Q_{1}^{\prime}$ with $e(\alpha)=i$ and $q \in \mathcal{P}(n \rightarrow s(\alpha))$. Thus $X_{\alpha}\left(x_{s(\alpha), \alpha}\right)=0$, for all $\alpha \in Q_{1}$ with $e(\alpha)=i$. This proves Claim 1 .

Claim 2: $X_{i} / \bigoplus_{\substack{\beta \in Q_{1} \\ e(\beta)=i}} \operatorname{Im} X_{\beta}$ is a Gorenstein-projective A-module for each $i \in Q_{0}$.

Indeed, since Coker $\phi$ is a Gorenstein-projective $\Lambda^{\prime}$-module, by the inductive hypothesis we know that

$$
\left(X_{i} / \bigoplus_{p \in \mathcal{P}(n \rightarrow i)} \operatorname{Im} X_{p}\right) / \bigoplus_{\substack{\alpha \in Q_{1}^{\prime} \\ e(\alpha)=i}} \operatorname{Im} \tilde{X}_{\alpha}
$$

is a Gorenstein-projective $A$-module: it is exactly the desired module by (5-1).

Now, (3) and Claim 1 mean that $X$ is a monic $\Lambda$-module; and (2), together with conclusion (i) of Lemma 5.3 and Claim 2, means that $X$ satisfies (G).

\section{Corollaries}

6A. For the definition of a Frobenius category in the sense of [Quillen 1973], we refer to [Happel 1988, p. 11; Keller 1990, Appendix A]. As a consequence of Theorem 5.1 and Proposition 2.4, we get the following characterization of self-injectivity.

Corollary 6.1. Let $A$ be a finite-dimensional algebra, and $Q$ a finite acyclic quiver. Then the following are equivalent:

(i) A is self-injective.

(ii) $\mathcal{G P}\left(A \otimes_{k} k Q\right)=\operatorname{Mon}(Q, A)$.

(iii) $\operatorname{Mon}(Q, A)$ is a Frobenius category.

Proof. (i) $\Longrightarrow$ (ii): If $A$ is self-injective, every $A$-module is Gorenstein-projective, and hence (ii) follows from Theorem 5.1. The implication (ii) $\Longrightarrow$ (iii) is well-known. 
(iii) $\Longrightarrow$ (i): Take a sink of $Q$, say vertex 1 , and consider $D\left(A_{A}\right) \otimes_{k} P(1)$. By Proposition 2.4 (iii) it is an injective object in $\operatorname{Mon}(Q, A)$, and hence, by assumption, it is a projective object in $\operatorname{Mon}(Q, A)$. By Proposition 2.4(ii) we know that $D\left(A_{A}\right)$, the first branch of $D\left(A_{A}\right) \otimes_{k} P(1)$, is a projective $A$-module, that is, $A$ is selfinjective.

Let $D^{b}(\Lambda)$ be the bounded derived category of $\Lambda$, and $K^{b}(\mathcal{P}(\Lambda))$ the bounded homotopy category of $\mathcal{P}(\Lambda)$. By definition the singularity category $D_{s g}^{b}(\Lambda)$ of $\Lambda$ is the Verdier quotient $D^{b}(\Lambda) / K^{b}(\mathcal{P}(\Lambda))$. Buchweitz [1987, Theorem 4.4.1] proved that if $\Lambda$ is Gorenstein, there is a triangle-equivalence $D_{s g}^{b}(\Lambda) \cong \underline{\mathcal{G P}(\Lambda)}$, where $\underline{\mathcal{G P}(\Lambda)}$ is the stable category of $\mathcal{G P}(\Lambda)$ modulo $\mathcal{P}(\Lambda)$; see also [Happel 1991, Theorem 4.6]. Note that if $A$ is Gorenstein, $\Lambda=A \otimes_{k} k Q$ is Gorenstein; see [Auslander and Reiten 1991b, Proposition 2.2]. So we have the following.

Corollary 6.2. Let $A$ be a finite-dimensional Gorenstein algebra, and $Q$ a finite acyclic quiver. Let $\Lambda=A \otimes_{k} k Q$. Then there is a triangle-equivalence $D_{s g}^{b}(\Lambda) \cong$ $\mathcal{G P}(\Lambda)$. In particular, if $A$ is self-injective, then there is a triangle-equivalence $D_{s g}^{b}(\Lambda) \cong \underline{\operatorname{Mon}(Q, A)}$.

6B. Recall the tensor product $Q \otimes Q^{\prime}$ of two finite quivers $Q$ and $Q^{\prime}$ (not necessarily acyclic). By definition $Q \otimes Q^{\prime}$ is the quiver with

$$
\left(Q \otimes Q^{\prime}\right)_{0}=Q_{0} \times Q_{0}^{\prime} \quad \text { and } \quad\left(Q \otimes Q^{\prime}\right)_{1}=\left(Q_{1} \times Q_{0}^{\prime}\right) \cup\left(Q_{0} \times Q_{1}^{\prime}\right) .
$$

More explicitly, if $\alpha: i \rightarrow j$ is an arrow of $Q$, then, for each vertex $t^{\prime} \in Q_{0}^{\prime}$, there is an arrow $\left(\alpha, t^{\prime}\right):\left(i, t^{\prime}\right) \rightarrow\left(j, t^{\prime}\right)$ of $Q \otimes Q^{\prime}$; and if $\beta^{\prime}: s^{\prime} \rightarrow t^{\prime}$ is an arrow of $Q^{\prime}$, then, for each vertex $i \in Q_{0}$, there is an arrow $\left(i, \beta^{\prime}\right):\left(i, s^{\prime}\right) \rightarrow\left(i, t^{\prime}\right)$ of $Q \otimes Q^{\prime}$.

Let $A=k Q / I$ and $B=k Q^{\prime} / I^{\prime}$ be two finite-dimensional $k$-algebras, where $Q$ and $Q^{\prime}$ are finite quivers (not necessarily acyclic), and $I, I^{\prime}$ are admissible ideals of $k Q, k Q^{\prime}$, respectively. Then

$$
A \otimes_{k} B \cong k\left(Q \otimes Q^{\prime}\right) / I \square I^{\prime},
$$

where $I \square I^{\prime}$ is the ideal of $k\left(Q \otimes Q^{\prime}\right)$ generated by $\left(I \times Q_{0}^{\prime}\right) \cup\left(Q_{0} \times I^{\prime}\right)$ and the elements

$$
\left(\alpha, t^{\prime}\right)\left(i, \beta^{\prime}\right)-\left(j, \beta^{\prime}\right)\left(\alpha, s^{\prime}\right)
$$

where $\alpha: i \rightarrow j$ is an arrow of $Q$, and $\beta^{\prime}: s^{\prime} \rightarrow t^{\prime}$ is an arrow of $Q^{\prime}$. See, for example, [Leszczyński 1994]. Note that $I \square I^{\prime}$ may not be zero even if $I=0=I^{\prime}$. We have proved this:

Fact. $A \otimes_{k} B$ is hereditary (that is, $I \square I^{\prime}=0$ ) if and only if either $A \cong k^{\left|Q_{0}\right|}$ as algebras and $I^{\prime}=0$, or $B \cong k^{\left|Q_{0}^{\prime}\right|}$ as algebras and $I=0$. 
6C. One can describe when $\Lambda$ is hereditary via $\operatorname{Mon}(Q, A)$.

Corollary 6.3. Let $A$ be a finite-dimensional basic algebra over an algebraically closed field $k, Q$ a finite acyclic quiver with $\left|Q_{1}\right| \neq 0$, and $\Lambda=A \otimes_{k} k Q$. Then $\mathcal{P}(\Lambda)=\operatorname{Mon}(Q, A)$ if and only if $\Lambda$ is hereditary.

Proof. Without loss of generality we may assume that $A$ is connected (an algebra is connected if it cannot be a product of two nonzero algebras).

If $\Lambda=A \otimes_{k} k Q$ is hereditary, then, by the fact above and the assumption on $Q$, we have $A=k$, and hence $\operatorname{Mon}(Q, k)=\mathcal{G P}(k Q)$ by Theorem 5.1. It follows that

$$
\operatorname{Mon}(Q, A)=\mathcal{G} \mathcal{P}(k Q)=\mathcal{P}(k Q)=\mathcal{P}(\Lambda) .
$$

Conversely, if $A \neq k, A$ is not semisimple since $A$ is assumed to be connected and basic and $k$ is assumed to be algebraically closed. It follows that there is a nonprojective $A$-module $M$. Take a sink of $Q$, say vertex 1 , and consider $\Lambda$-module $X=M \otimes_{k} P(1)$, where $P(1)$ is the simple projective $k Q$-module at vertex 1 . Then $X \in \operatorname{Mon}(Q, A)$, but $X \notin \mathcal{P}(\Lambda)$.

\section{References}

[Arnold 2000] D. M. Arnold, Abelian groups and representations of finite partially ordered sets, CMS Books in Mathematics 2, Springer, New York, 2000. MR 2001g:16030 Zbl 0959.16011

[Auslander and Bridger 1969] M. Auslander and M. Bridger, Stable module theory, Memoirs of the American Mathematical Society 94, American Mathematical Society, Providence, R.I., 1969. MR 42 \#4580 Zbl 0204.36402

[Auslander and Reiten 1991a] M. Auslander and I. Reiten, "Applications of contravariantly finite subcategories”, Adv. Math. 86:1 (1991), 111-152. MR 92e:16009 Zbl 0774.16006

[Auslander and Reiten 1991b] M. Auslander and I. Reiten, "Cohen-Macaulay and Gorenstein Artin algebras", pp. 221-245 in Representation theory of finite groups and finite-dimensional algebras (Bielefeld, 1991), edited by G. O. Michler and C. M. Ringel, Progr. Math. 95, Birkhäuser, Basel, 1991. MR 92k:16018 Zbl 0776.16003

[Auslander and Smalø 1981] M. Auslander and S. O. Smalø, "Almost split sequences in subcategories”, J. Algebra 69:2 (1981), 426-454. MR 82j:16048a Zbl 0457.16017

[Auslander et al. 1995] M. Auslander, I. Reiten, and S. O., Representation theory of Artin algebras, Cambridge Studies in Advanced Mathematics 36, Cambridge University Press, 1995. MR 96c: 16015 Zbl 0834.16001

[Avramov and Martsinkovsky 2002] L. L. Avramov and A. Martsinkovsky, "Absolute, relative, and Tate cohomology of modules of finite Gorenstein dimension", Proc. London Math. Soc. (3) 85:2 (2002), 393-440. MR 2003g:16009 Zbl 1047.16002

[Beligiannis 2005] A. Beligiannis, "Cohen-Macaulay modules, (co)torsion pairs and virtually Gorenstein algebras”, J. Algebra 288:1 (2005), 137-211. MR 2006i:16017 Zbl 1119.16007

[Buchweitz 1987] R.-O. Buchweitz, "Maximal Cohen-Macaulay modules and Tate cohomology over Gorenstein rings", Unpub. manu., 1987, Available at http://tinyurl.com/cohen-mac1986-pdf.

[Chen 2011] X.-W. Chen, "The stable monomorphism category of a Frobenius category", Math. Res. Lett. 18:1 (2011), 125-137. MR 2012e:18024 Zbl 06026606 
[Enochs and Jenda 1995] E. E. Enochs and O. M. G. Jenda, "Gorenstein injective and projective modules", Math. Z. 220:4 (1995), 611-633. MR 97c:16011 Zbl 0845.16005

[Enochs and Jenda 2000] E. E. Enochs and O. M. G. Jenda, Relative homological algebra, de Gruyter Expositions in Mathematics 30, Walter de Gruyter, Berlin, 2000. MR 2001h:16013 Zbl 0952.13001

[Gao and Zhang 2010] N. Gao and P. Zhang, "Gorenstein derived categories", J. Algebra 323:7 (2010), 2041-2057. MR 2011f:18017 Zbl 1222.18005

[Happel 1988] D. Happel, Triangulated categories in the representation theory of finite-dimensional algebras, London Mathematical Society Lecture Note Series 119, Cambridge University Press, 1988. MR 89e:16035 Zbl 0635.16017

[Happel 1991] D. Happel, “On Gorenstein algebras”, pp. 389-404 in Representation theory of finite groups and finite-dimensional algebras (Bielefeld, 1991), edited by G. O. Michler and C. M. Ringel, Progr. Math. 95, Birkhäuser, Basel, 1991. MR 92k:16022 Zbl 0759.16007

[Holm 2004] H. Holm, “Gorenstein homological dimensions", J. Pure Appl. Algebra 189:1-3 (2004), 167-193. MR 2004k:16013 Zbl 1050.16003

[Iyama et al. 2011] O. Iyama, K. Kato, and J.-I. Miyachi, "Recollement of homotopy categories and Cohen-Macaulay modules", J. K-Theory 8:3 (2011), 507-542. MR 2012k:18018 Zbl 1251.18008

[Keller 1990] B. Keller, “Chain complexes and stable categories”, Manuscripta Math. 67:4 (1990), 379-417. MR 91h:18006 Zbl 0753.18005

[Krause and Solberg 2003] H. Krause and Ø. Solberg, "Applications of cotorsion pairs", J. London Math. Soc. (2) 68:3 (2003), 631-650. MR 2004k:16028 Zbl 1061.16006

[Kussin et al. 2012] D. Kussin, H. Lenzing, and H. Meltzer, "Nilpotent operators and weighted projective lines", 2012. To appear in J. Reine Angew. Math. arXiv 1002.3797

[Leszczyński 1994] Z. Leszczyński, “On the representation type of tensor product algebras", Fund. Math. 144:2 (1994), 143-161. MR 95b:16013 Zbl 0817.16008

[Li and Zhang 2010] Z.-W. Li and P. Zhang, "A construction of Gorenstein-projective modules", J. Algebra 323:6 (2010), 1802-1812. MR 2011d:16012 Zbl 1210.16011

[Moore 2010] A. Moore, "The Auslander and Ringel-Tachikawa theorem for submodule embeddings", Comm. Algebra 38:10 (2010), 3805-3820. MR 2011k:16040 Zbl 1237.16015

[Quillen 1973] D. Quillen, "Higher algebraic K-theory, I", pp. 85-147 in Algebraic K-theory, I: Higher K-theories (Seattle, WA, 1972), edited by H. Bass, Lecture Notes in Math. 341, Springer, Berlin, 1973. MR 49 \#2895 Zbl 0292.18004

[Ringel 1984] C. M. Ringel, Tame algebras and integral quadratic forms, Lecture Notes in Mathematics 1099, Springer, Berlin, 1984. MR 87f:16027 Zbl 0546.16013

[Ringel and Schmidmeier 2006] C. M. Ringel and M. Schmidmeier, "Submodule categories of wild representation type”, J. Pure Appl. Algebra 205:2 (2006), 412-422. MR 2006i:16025 Zbl 1147. 16019

[Ringel and Schmidmeier 2008a] C. M. Ringel and M. Schmidmeier, "The Auslander-Reiten translation in submodule categories", Trans. Amer. Math. Soc. 360:2 (2008), 691-716. MR 2008j:16058 Zbl 1154.16011

[Ringel and Schmidmeier 2008b] C. M. Ringel and M. Schmidmeier, "Invariant subspaces of nilpotent linear operators, I", J. Reine Angew. Math. 614 (2008), 1-52. MR 2009d:16016 Zbl 1145.16005

[Ringel and Zhang 2011] C. M. Ringel and P. Zhang, "Representations of quivers over the algebras of dual numbers", preprint, 2011. arXiv 1112.1924

[Simson 2007] D. Simson, "Representation types of the category of subprojective representations of a finite poset over $K[t] /\left(t^{m}\right)$ and a solution of a Birkhoff type problem”, J. Algebra 311:1 (2007), 1-30. MR 2009b:16040 Zbl 1123.16010 
[Xiong and Zhang 2012] B.-L. Xiong and P. Zhang, "Gorenstein-projective modules over triangular matrix Artin algebras", J. Algebra Appl. 11:4 (2012), Article ID 1250066, 14. MR 2959415 Zbl pre06078475

[Zhang 2011] P. Zhang, "Monomorphism categories, cotilting theory, and Gorenstein-projective modules", J. Algebra 339 (2011), 181-202. MR 2012k:16034 Zbl pre06009303

Received May 10, 2012.

Xiu-Hua Luo

DEPARTMENT OF MATHEMATICS

SHANGHAi JiAO TONG UNIVERSITY

DONGCHUAN ROAD 800

SHANGHAI, 200240

CHINA

x.h.luo@sjtu.edu.cn

PU ZHANG

DEPARTMENT OF MATHEMATICS

SHANGHAi JiaO TONG UNIVERSITY

DONGCHUAN ROAD 800

ShanghaI, 200240

CHINA

pzhang@sjtu.edu.cn 


\title{
PACIFIC JOURNAL OF MATHEMATICS
}

\author{
msp.org/pjm
}

Founded in 1951 by E. F. Beckenbach (1906-1982) and F. Wolf (1904-1989)

\section{EDITORS}

V. S. Varadarajan (Managing Editor)

Department of Mathematics

University of California

Los Angeles, CA 90095-1555

pacific@math.ucla.edu

Paul Balmer

Department of Mathematics

University of California

Los Angeles, CA 90095-1555

balmer@math.ucla.edu

Daryl Cooper

Department of Mathematics

University of California

Santa Barbara, CA 93106-3080 cooper@math.ucsb.edu

Jiang-Hua $\mathrm{Lu}$

Department of Mathematics

The University of Hong Kong

Pokfulam Rd., Hong Kong jhlu@maths.hku.hk
Don Blasius

Department of Mathematics University of California

Los Angeles, CA 90095-1555

blasius@math.ucla.edu

Robert Finn

Department of Mathematics Stanford University

Stanford, CA 94305-2125

finn@math.stanford.edu

Sorin Popa

Department of Mathematics

University of California

Los Angeles, CA 90095-1555

popa@math.ucla.edu

Paul Yang

Department of Mathematics

Princeton University

Princeton NJ 08544-1000

yang@math.princeton.edu

\section{PRODUCTION}

Silvio Levy, Scientific Editor, production@msp.org

\section{SUPPORTING INSTITUTIONS}

ACADEMIA SINICA, TAIPEI

CALIFORNIA INST. OF TECHNOLOGY

INST. DE MATEMÁTICA PURA E APLICADA

KEIO UNIVERSITY

MATH. SCIENCES RESEARCH INSTITUTE

NEW MEXICO STATE UNIV.

OREGON STATE UNIV.

\author{
STANFORD UNIVERSITY \\ UNIV. OF BRITISH COLUMBIA \\ UNIV. OF CALIFORNIA, BERKELEY \\ UNIV. OF CALIFORNIA, DAVIS \\ UNIV. OF CALIFORNIA, LOS ANGELES \\ UNIV. OF CALIFORNIA, RIVERSIDE \\ UNIV. OF CALIFORNIA, SAN DIEGO \\ UNIV. OF CALIF., SANTA BARBARA
}

\author{
Vyjayanthi Chari \\ Department of Mathematics \\ University of California \\ Riverside, CA 92521-0135 \\ chari@math.ucr.edu \\ Kefeng Liu \\ Department of Mathematics \\ University of California \\ Los Angeles, CA 90095-1555 \\ liu@math.ucla.edu \\ Jie Qing \\ Department of Mathematics \\ University of California \\ Santa Cruz, CA 95064 \\ qing@cats.ucsc.edu
}

These supporting institutions contribute to the cost of publication of this Journal, but they are not owners or publishers and have no responsibility for its contents or policies.

See inside back cover or msp.org/pjm for submission instructions.

The subscription price for 2013 is US \$400/year for the electronic version, and \$485/year for print and electronic.

Subscriptions, requests for back issues and changes of subscribers address should be sent to Pacific Journal of Mathematics, P.O. Box 4163, Berkeley, CA 94704-0163, U.S.A. The Pacific Journal of Mathematics is indexed by Mathematical Reviews, Zentralblatt MATH, PASCAL CNRS Index, Referativnyi Zhurnal, Current Mathematical Publications and the Science Citation Index.

The Pacific Journal of Mathematics (ISSN 0030-8730) at the University of California, c/o Department of Mathematics, 798 Evans Hall \#3840, Berkeley, CA 94720-3840, is published monthly except July and August. Periodical rate postage paid at Berkeley, CA 94704, and additional mailing offices. POSTMASTER: send address changes to Pacific Journal of Mathematics, P.O. Box 4163, Berkeley, CA 94704-0163.

PJM peer review and production are managed by EditFLOW ${ }^{\circledR}$ from Mathematical Sciences Publishers.

PUBLISHED BY

mathematical sciences publishers

nonprofit scientific publishing

http://msp.org/

(C) 2013 Mathematical Sciences Publishers 


\section{PACIFIC JOURNAL OF MATHEMATICS}

Volume $264 \quad$ No. $1 \quad$ July 2013

On the center of fusion categories

ALAIN BRUGUIÈRES and ALEXIS VIRELIZIER

Connected quandles associated with pointed abelian groups

W. EdWin Clark, MoHamed ElHamdadi, Xiang-DONG HoU,

MASAHICO SAITO and TIMOTHY YEATMAN

Entropy and lowest eigenvalue on evolving manifolds

HongXin GuO, ROBERT PHILIPOWSKI and ANTON THALMAIER

Poles of certain residual Eisenstein series of classical groups

DIHUA JIANG, BAIYING LIU and LEI ZHANG

Harmonic maps on domains with piecewise Lipschitz continuous metrics 125

HAIGANG LI and CHANGYOU WANG

$q$-hypergeometric double sums as mock theta functions

JEREMY LOVEJOY and ROBERT OSBURN

Monic representations and Gorenstein-projective modules

XIU-HuA LUO and PU ZHANG

Helicoidal flat surfaces in hyperbolic 3-space

Antonio Martínez, JoÃo Paulo dos Santos and Keti

TENENBLAT

On a Galois connection between the subfield lattice and the multiplicative subgroup lattice

JOHN K. MCVEY

Some characterizations of Campanato spaces via commutators on Morrey 221 spaces

Shaoguang Shi and Shanzhen Lu

The Siegel-Weil formula for unitary groups 